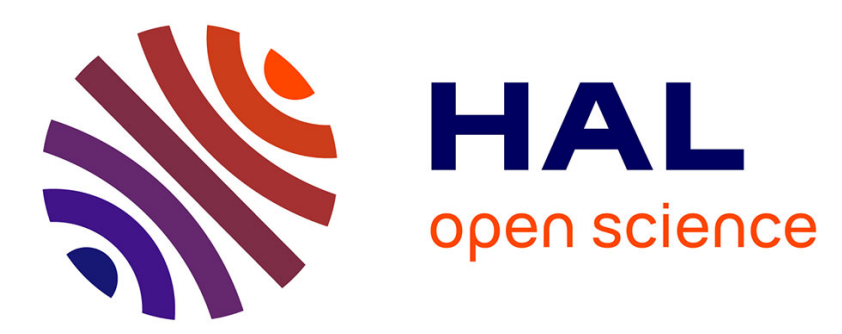

\title{
Preparation of Vinylcyclopropanes by Sodium Mediated Reductive Isomerization of Methylenecyclopropanes
}

\author{
Karel Le Jeune, Sabine Chevallier-Michaud, David Gatineau, Laurent
}

Giordano, Alphonse Tenaglia, Hervé Clavier

\section{- To cite this version:}

Karel Le Jeune, Sabine Chevallier-Michaud, David Gatineau, Laurent Giordano, Alphonse Tenaglia, et al. Preparation of Vinylcyclopropanes by Sodium Mediated Reductive Isomerization of Methylenecyclopropanes. Journal of Organic Chemistry, 2015, 80 (17), pp.8821-8829. 10.1021/acs.joc.5b01205 . hal-01409710

\author{
HAL Id: hal-01409710 \\ https://hal.science/hal-01409710
}

Submitted on 28 Dec 2021

HAL is a multi-disciplinary open access archive for the deposit and dissemination of scientific research documents, whether they are published or not. The documents may come from teaching and research institutions in France or abroad, or from public or private research centers.
L'archive ouverte pluridisciplinaire $\mathbf{H A L}$, est destinée au dépôt et à la diffusion de documents scientifiques de niveau recherche, publiés ou non, émanant des établissements d'enseignement et de recherche français ou étrangers, des laboratoires publics ou privés. 


\title{
Preparation of Vinylcyclopropanes by Sodium Mediated Reductive Isomerization of Methylenecyclopropanes
}

\author{
Karel Le Jeune, Sabine Chevallier-Michaud, David Gatineau, Laurent Giordano, Alphonse Tenaglia, \\ and Hervé Clavier*
}

Aix Marseille Université, Centrale Marseille, CNRS, iSm2 UMR 7313, 13397 Marseille, France

Supporting Information

\begin{abstract}
We disclosed therein a new reaction of reductive isomerization of methylenecyclopropanes (MCPs) to vinylcyclopropanes (VCPs). On treatment with sodium metal in liquid ammonia, MCPs bearing a $\mathrm{C}-\mathrm{O}$ bond at allylic position undergo both a reductive cleavage of the $\mathrm{C}-\mathrm{O}$ bond

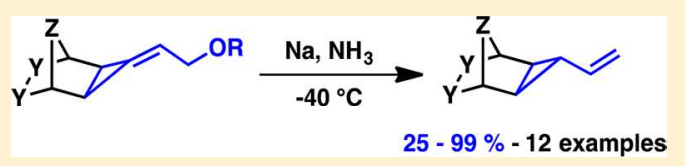
and an isomerization of the $\mathrm{C}-\mathrm{C}$ double bond giving rise to VCPs. The scope of the reductive isomerization was investigated and showed a broad applicability since various functional groups are tolerated. MCP substrates were straightforwardly prepared by a palladium-promoted $[2+1]$ cycloaddition between norbornene derivatives with alkynes.
\end{abstract}

$\mathrm{V}$ inylcyclopropanes (VCPs) are versatile synthons in various organic transformations, ${ }^{1}$ mainly cycloadditions, as well as important structural motifs in natural product ${ }^{2}$ such as chrysanthemic acid, dictyopterenes, ambruticin $S$ or lindenane-type sesquiterpenoids. Therefore, their synthesis has attracted much attention and has been translated into the development of several methodologies involving the formation of cyclopropane ring: cyclopropanations between either a diazo compound and a 1,4 diene or an allylic diazo compound and an alkene, ${ }^{3}$ ring rearrangement, ${ }^{4}$ Michael addition or substitution. ${ }^{5}$ Vinylcyclopropanes can be also prepared by functional group transformations from cyclopropyl precursors. $^{6}$ In 1982, Catellani and Chiusoli reported a palladiumpromoted vinylcyclopropane formation starting from norbornene and 1-bromo-1-octene (Scheme 1)..$^{7}$ The reaction occurring by two consecutive insertions of palladium species and subsequent $\beta-\mathrm{H}$ elimination was unfortunately not exemplified. Alternatively, Murakami ${ }^{8}$ and Lautens ${ }^{9}$ reported independently an interesting rhodium-promoted vinylcyclopropanation of strained olefins using vinyl boronate derivatives. Herein, we report a new approach for the preparation of the VCP substructure, which consists in a two steps procedure: a $[2+1]$ cycloaddition followed by a reductive isomerization (Scheme 1).

In 2011, we disclosed an unprecedented intermolecular tandem $[2+1] /[3+2]$ cycloaddition sequence catalyzed by platinum-phosphinito-phosphinous acid complexes 3 between norbornadiene and alkynes giving rise to tricyclic compounds 2. ${ }^{10}$ Further investigations showed that the reaction occurred in two steps (Scheme 2). First, a $[2+1]$ cycloaddition promoted by catalyst $3,{ }^{11}$ or palladium analogues in situ generated from $\mathrm{Pd}(\mathrm{OAc})_{2}$ and secondary phosphine oxides, afforded methylenecyclopropanes (MCPs) 1 . Then, platinumbased complex 3 was able to achieve the diastereoselective [3 + 2] cycloaddition between 1a and another alkyne partner with the proviso that MCPs 1 bear an oxygen substituent on the propargylic carbon atom. Whereas these $[2+1]$ cycloadditions have been well studied in our laboratories ${ }^{12}$ and by others, ${ }^{13}$ the platinum-promoted $[3+2]$ cycloaddition has not been further investigated so far. ${ }^{14}$

To broaden the scope of this reaction, we wondered whether we could achieve the $[3+2]$ cycloaddition in absence of the bicyclo[2.2.1] heptane moiety linked to the MCP. The ring strain of the norbornene pattern might enhance the reactivity of MCP derivatives. To that end, we planned to prepare suitable substrates by a $[2+1]$ cycloaddition of diazabicyclic alkene and propargyl acetate to afford quantitatively the adduct $\mathbf{1 b}$ and attempted the reductive cleavage of the $\mathrm{N}-\mathrm{N}$ bond. Following the reported conditions for the reductive $\mathrm{N}-\mathrm{N}$ cleavage of hydrazines, ${ }^{15,16}$ cyclic hydrazine $\mathbf{1 b}$ was treated with 2.5 equiv of sodium in liquid ammonia and no traces of the expected product $4 \mathrm{~b}$ could be detected (Scheme 3). A new compound was isolated and identified as $\mathbf{5 b}$. Its formation resulted from a reductive isomerization of the MCP with release of the acetoxy group by $\mathrm{C}-\mathrm{O}$ bond cleavage. ${ }^{17}$ Of note, $\mathbf{5 b}$ was obtained as a single diastereomer, with the vinyl substituent trans to other substituents of the cyclopropane. Increasing the quantity of sodium to 5 equiv led effectively to the cleavage of the $\mathrm{N}-\mathrm{N}$ bond, which is subsequent to the formation of the VCP since compound $\mathbf{6 b}$ was isolated in $55 \%$. Single-crystal X-ray analysis of $\mathbf{6 b}$ unambiguously confirmed the atom connectivity and the relative stereochemistry of all substituents (Figure 1).

This methodology represents an interesting alternative to rhodium-promoted vinylcyclopropanation of olefins developed by Murakami ${ }^{8}$ and Lautens; ${ }^{9}$ therefore, we decided to pursue the investigation of this original reactivity of MCPs. ${ }^{18} \mathrm{We}$ started by the preparation of a wide range of $[2+1]$ cycloadducts through the reaction of norbornene derivatives 
Scheme 1. Preparation of Vinylcyclopropanes for Norbornene Derivatives

$$
\text { Catellani \& Chiusoli }
$$
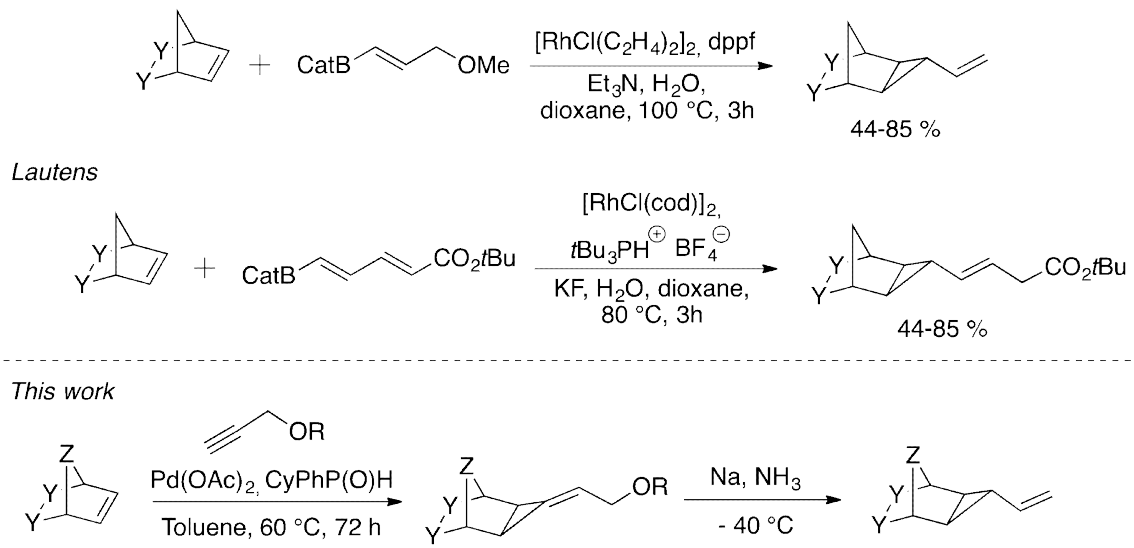

Scheme 2. Platinum-Catalyzed $[3+2]$ Cycloadditions

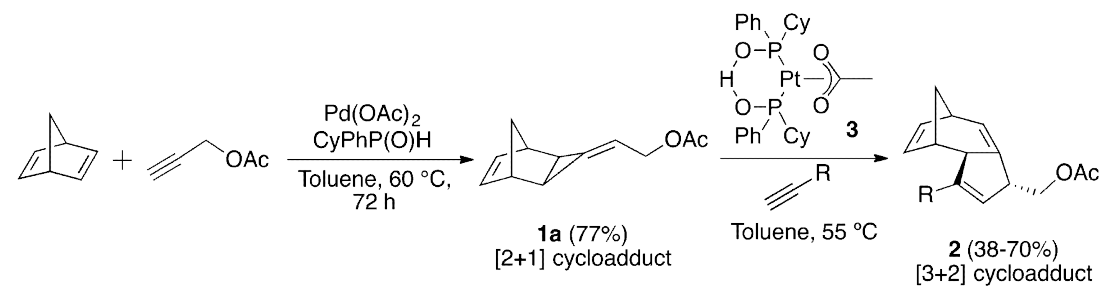

Scheme 3. Reductive N-N Cleavage of Cyclic Hydrazine $1 \mathrm{~b}$

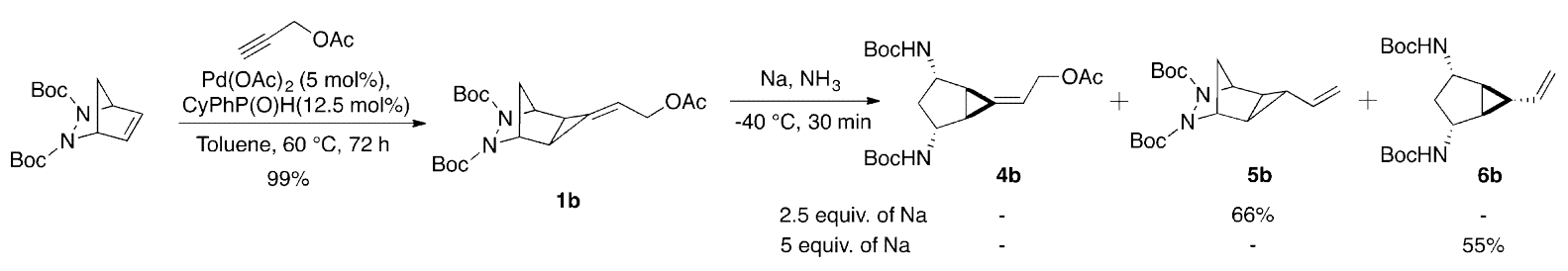

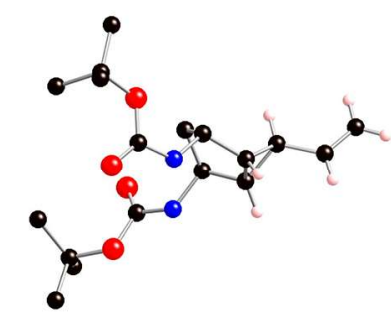

Figure 1. Ball-and-stick representation of vinylcyclopropane $\mathbf{6 b}$ obtained by X-ray analysis (most of the hydrogen atoms have been omitted for clarity).

with alkynes catalyzed by $\operatorname{Pd}(\mathrm{OAc})_{2}$ in association with secondary phosphine oxides $(\operatorname{PhCyP}(\mathrm{O}) \mathrm{H})$ (Table 1$)$. Various functional groups are well tolerated and, in a general manner, good yields were obtained with the exception of cycloadduct If (entry 4 ). Of note, due to the $E / Z$ isomerism of the $\mathrm{C}-\mathrm{C}$ double bond and the presence of a stereogenic center, $\mathbf{l h}$ was isolated as an inseparable 1:1 mixture of two diastereomers (entry 6).
We then examined several reducing agents and reaction conditions using the benchmark substrate $\mathbf{1 d}$ (Table 2). With the use of sodium in liquid ammonia at $-40{ }^{\circ} \mathrm{C}$, the expected reduced product $\mathbf{5 d}$ was isolated in an excellent yield and a good diastereoselectivity since small amounts of the cyclopropane with a cis arrangement were detected (entry 1). When the reaction was performed under the reaction conditions of the Bouveault-Blanc reduction, ${ }^{19}$ with absolute ethanol as proton source, only a low yield in $\mathbf{5 d}$ was obtained (entry 2). The major product isolated is the alcohol 1 n resulting from the reduction of the ester function. The use of sodium naphthalenide ${ }^{20}$ in THF at room temperature led to the selective formation of alcohol $\mathbf{1 n}$ in quantitative yield (entry 3). Samarium(II) iodide was also tested but in absence of a protic solvent no reaction occurred, and with methanol a low yield in 1n was obtained (entries 4 and 5).

Having established the optimal reaction conditions, we further investigated the scope of reaction with a range of ethylidenecyclopropanes bearing an acetate group (Table 3). Bicyclic hydrazines $1 \mathbf{b}$ and $\mathbf{1 c}$ gave the corresponding VCP derivatives with moderate yields but an excellent diastereoselectivity (entries 1 and 2 ). With the substrate 1e, a 
Table 1. Preparation of Alkylidenecyclopropanes by $[2+1]$ Cycloaddition $^{a}$

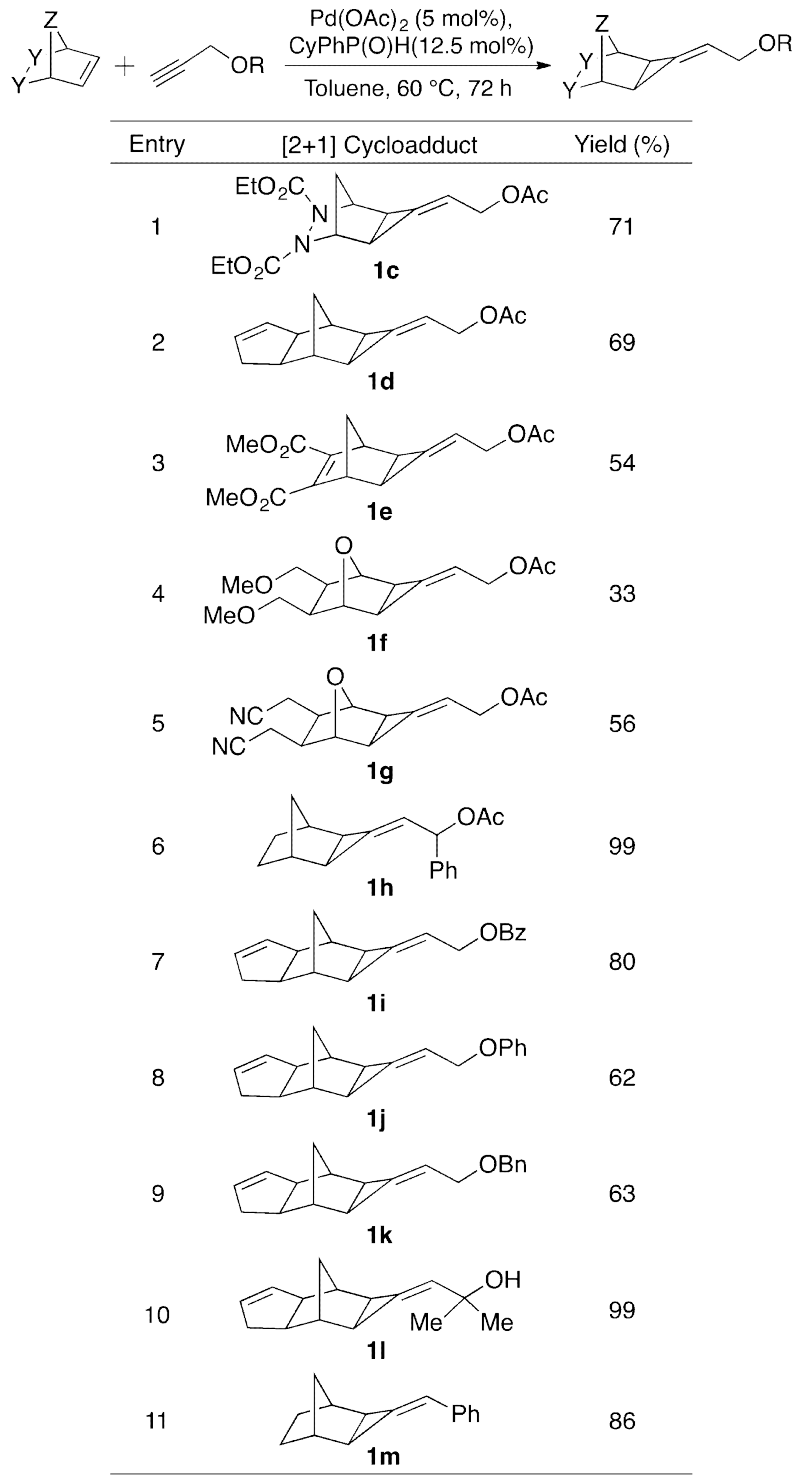

${ }^{a}$ Reaction conditions: norbornene derivative/alkyne $1: 3, \mathrm{Pd}(\mathrm{OAc})_{2}(5$ $\mathrm{mol} \%), \mathrm{CyPhP}(\mathrm{O}) \mathrm{H}(12.5 \mathrm{~mol} \%)$, toluene, $60{ }^{\circ} \mathrm{C}, 72 \mathrm{~h}$.

complex mixture was obtained, probably due to the competitive reduction of the norbornene double bond (entry 4). ${ }^{21}$ To our delight, VCP 1o, prepared through chemoselective epoxidation of $1 \mathbf{a}^{22}$ was quantitatively converted to VCP 5o with high diastereoselectivity (entry 5 ). The reductive isomerization was found also tolerant to other ether functions such as the 7-oxa bicyclo[2.2.1] heptane skeleton (entry 6). However, the presence of cyano groups decreased significantly both yield and diastereoselectivity (entry 7). This might be the result of competitive cyano groups reduction. Despite a good selectivity, with the phenylsubstituted compound $\mathbf{1 h}$, only a low yield could be reached due to the formation of the over-reduced product $7 \mathrm{~h}$ in $62 \%$ yield (entry 8 ). Unfortunately, it was not possible to separate 5h from 7 h by silica gel chromatography.

We then studied the influence of the nature of the allylic substituent on the transformation outcome (Table 4). The replacement of the acetate group with a benzoate led to diminish slightly both yield and selectivity and required a 2fold excess of sodium metal (entries 1 and 2). In addition to carboxylates, the phenoxy group was found to be effective (entry 3 ). With the benzyl ether substrate $1 \mathbf{k}$, only the deprotection delivering alcohol 1 n was observed (entry 4). Several alcohols have been also tested and satisfactorily corresponding VCPs have been isolated in moderate yields and modest to good diastereoselectivities (entries 5-7). This includes the gem-dimethyl substituted alcohol 11 that, with 6 equiv of sodium, gave rise to the 2-methyl-propen-1-ylcyclopropane 51. The modest yields obtained with 1 n and 11 (entries 5 and 6) can be explained by the fact that, even with 6 equiv of sodium, reactions did not reach completion and starting material alcohols were recovered. This suggests that the alkoxide formation is competing pathway to the reductive isomerization. In absence of the allylic substituent, such as for the benzylidenecyclopropane $\mathbf{1 m}$, only the fully diastereoselective reduction the $\mathrm{C}-\mathrm{C}$ double bond occurred leaving the cyclopropane ring intact (entry 8 ).

As depicted in Scheme 4, we assumed that the reaction mechanism involves a first single-electron transfer to generate the radical anion intermediate $\mathbf{B}$. An elimination of the acetate group leads to the formation of vinyl species $\mathbf{C}$ and a second electron transfer gives $\mathbf{D}$, which upon protonolysis releases the VCP E.

In summary, we developed a new transformation allowing the formation of vinylcyclopropanes from MCPs. The treatment of the ethylidenecyclopropanes bearing an allylic group with sodium metal in liquid ammonia led to a reductive isomerization and generated VCPs with moderate to quantitative both yields and diastereoselectivities. This reaction was found tolerant to various function groups to the exception of those highly reducible. We are now exploring the reactivity of the VCP derivatives.

Table 2. Investigation of the Reducing Agent

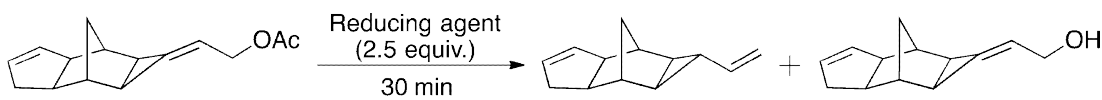

10

$1 n$

\begin{tabular}{|c|c|c|c|c|}
\hline \multirow[b]{2}{*}{ entry } & \multirow[b]{2}{*}{ reducing agent } & \multirow[b]{2}{*}{ conditions } & \multicolumn{2}{|c|}{ yield (\%) } \\
\hline & & & $5 d$ (d.r.) & 1n \\
\hline 1 & $\mathrm{Na}$ & $\mathrm{NH}_{3},-40{ }^{\circ} \mathrm{C}$ & $86(8.5: 1)$ & - \\
\hline 2 & $\mathrm{Na}$ & $\mathrm{NH}_{3}, \mathrm{EtOH}, 25^{\circ} \mathrm{C}$ & $30(1: 0)$ & 67 \\
\hline 3 & $\mathrm{Na}^{+} \mathrm{C}_{10} \mathrm{H}_{8}^{-\bullet}$ & THF, $25{ }^{\circ} \mathrm{C}$ & - & 98 \\
\hline 4 & $\mathrm{Sml}_{2}$ & THF, $25^{\circ} \mathrm{C}$ & - & - \\
\hline 5 & $\mathrm{Sml}_{2}$ & $\mathrm{THF}, \mathrm{MeOH}, 25^{\circ} \mathrm{C}$ & - & 22 \\
\hline
\end{tabular}


Table 3. Reductive Isomerization with a Variety of $\mathrm{MCPs}^{a}$

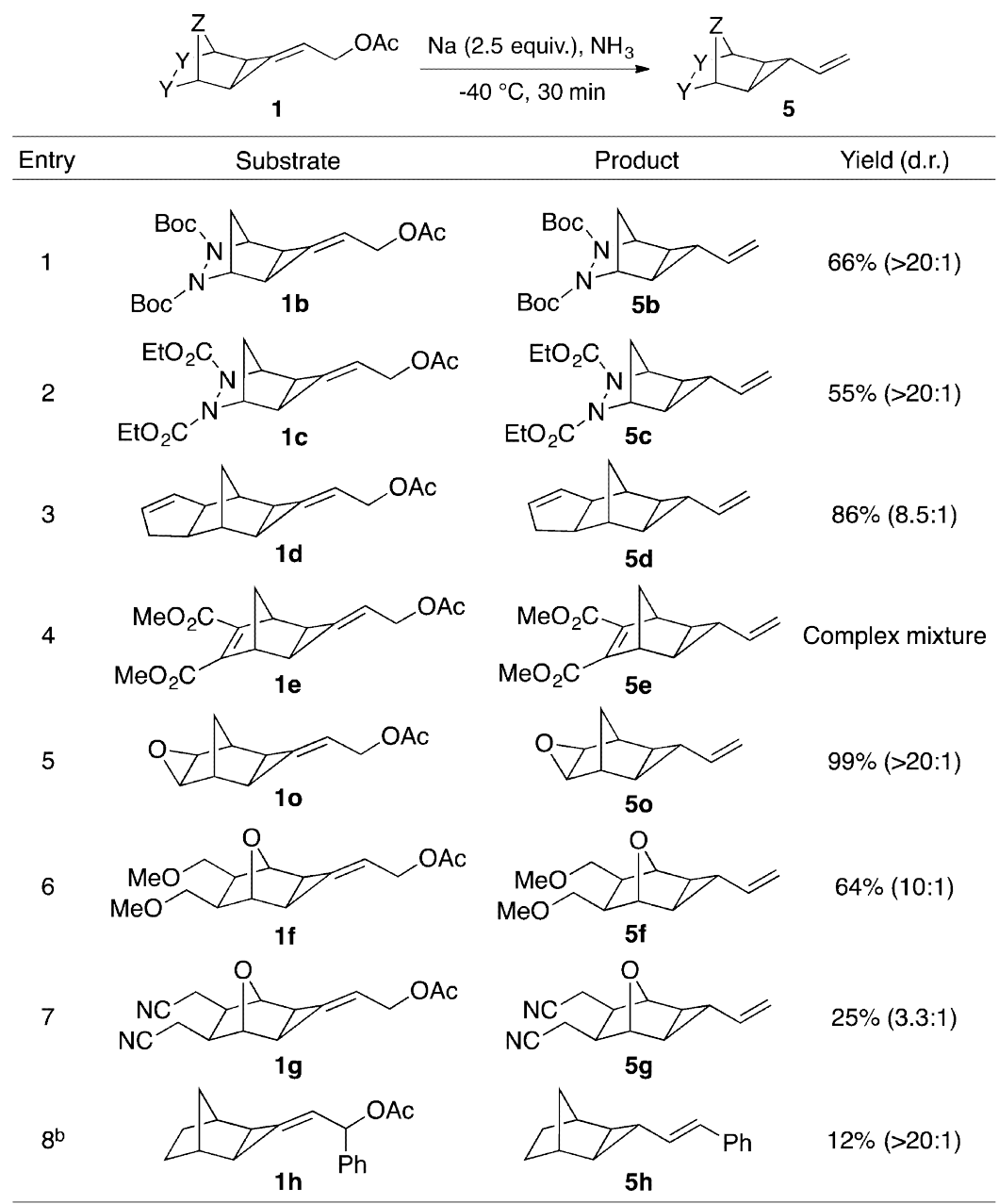

${ }^{a}$ Reaction conditions: substrate $(0.5 \mathrm{mmol}), \mathrm{Na}\left(29 \mathrm{mg}, 1.25 \mathrm{mmol}, 2.5\right.$ equiv), $\mathrm{NH}_{3}(20 \mathrm{~mL}) .{ }^{b} 62 \%$ of over-reduced compound $7 \mathrm{~h}$ was also isolated as unseparable mixture with $\mathbf{5 h}$.

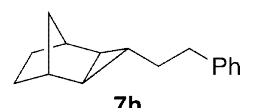

7h

\section{EXPERIMENTAL SECTION}

General Considerations. All reagents were obtained from commercial sources and used as received. Solvents (THF, DCM, toluene, and $\mathrm{Et}_{2} \mathrm{O}$ ) were purified and dried over solvent purification system or dried by standard procedures prior to use. ${ }^{23}$ Petroleum ether (PE) (fraction between 40 and $60{ }^{\circ} \mathrm{C}$ ) and ethyl acetate used for chromatographic separation were used as technical grade. Unless otherwise stated, all reactions were carried out in an atmosphere of dry nitrogen or argon using oven-dried $\left(120{ }^{\circ} \mathrm{C}\right)$ glasswares. Analytical TLCs were performed on ready-made plates coated with silica gel on aluminum. Products were visualized by ultraviolet light and treatment either with $p$-anisaldehyde or phosphomolybdic acid stain followed by gentle heating or with iodine stain. Flash chromatography was performed using silica gel $60 \quad(230-400$ mesh). ${ }^{1} \mathrm{H}$ and ${ }^{13} \mathrm{C}$, NMR spectra were recorded in $\mathrm{CDCl}_{3}$ at ambient temperature on spectrometers operating at 300 or $400 \mathrm{MHz}$ for ${ }^{1} \mathrm{H} .{ }^{13} \mathrm{C}$ nucleus was observed with ${ }^{1} \mathrm{H}$ decoupling. Solvent residual signals were used as internal standard. ${ }^{24}$ Chemical shifts $(\delta)$ and coupling constants $(J)$ are given in ppm and $\mathrm{Hz}$, respectively. The peaks patterns are indicated as the following format multiplicity (s, singlet; d, doublet; $\mathrm{t}$, triplet; $\mathrm{q}$, quartet; sept, septuplet; $\mathrm{m}$, multiplet; $\mathrm{dd}$, doublet of doublet; $\mathrm{dt}$, doublet of triplet; $\mathrm{dm}$, doublet of multiplet, etc.). The prefix br. indicates a broadened signal. HRMS were recorded on spectrometers equipped with an Atmospheric
Pressure Ionization (API) source. Mass spectra were obtained a Time Of Flight (TOF) analyzer. X-ray Diffraction: intensity data were collected on a diffractometer using Mo $\mathrm{K} \alpha$ radiation $(0.71073$ $\AA$ ) at 293(2) K. The CIF file of compound $\mathbf{6 b}$ has been deposited with the CCDC number 1048899.

General Procedure A: Palladium-Catalyzed $[2+1]$ Cycloaddition. In a flame-dried Schlenck, $\mathrm{Pd}(\mathrm{OAc})_{2}(5 \mathrm{~mol} \%)$ and $\mathrm{PhCyP}(\mathrm{O}) \mathrm{H}(12.5 \mathrm{~mol} \%)$ were introduced under argon and dissolved in dry and degassed toluene. The resulting yellow solution was stirred at $60{ }^{\circ} \mathrm{C}$ for $30 \mathrm{~min}$; the yellow color disappeared. Then, the norbornene derivative, alkyne ( 3 equiv), and dry and degassed toluene were added. The resulting mixture was stirred at $60{ }^{\circ} \mathrm{C}$ for $72 \mathrm{~h}$. Then, the volatiles were removed under reduced pressure. The crude product was purified on silica gel chromatography to obtain the desired product.

$\left(1 R^{*}, 4 S^{*}, 5 S^{*}\right)$-Tricyclo[3.2.1.0 $0^{2,4}$ oct-6-en-3-ylidene)ethyl Acetate $1 a^{11}$ Following the general procedure A, after purification on silica gel chromatography, the product was obtained as a colorless oil ( 653 $\mathrm{mg}, 77 \%$ yield). ${ }^{1} \mathrm{H}$ NMR $\left(400 \mathrm{MHz}, \mathrm{CDCl}_{3}\right): \delta=6.36(\mathrm{br} \mathrm{s}, 2 \mathrm{H})$, $5.74(\mathrm{tt}, J=1.1$ and $6.5 \mathrm{~Hz}, 1 \mathrm{H}), 4.60-4.70(\mathrm{~m}, 2 \mathrm{H}), 3.03(\mathrm{~d}, J=$ $6.0 \mathrm{~Hz}, 2 \mathrm{H}), 2.05(\mathrm{~s}, 3 \mathrm{H}), 1.57(\mathrm{br} \mathrm{s}, 2 \mathrm{H}), 1.03(\mathrm{~d}, J=8.5 \mathrm{~Hz}, 1 \mathrm{H})$, $0.91(\mathrm{~d}, J=8.5 \mathrm{~Hz}, 1 \mathrm{H}) .{ }^{13} \mathrm{C}$ NMR $\left(100 \mathrm{MHz}, \mathrm{CDCl}_{3}\right): \delta=170.9$ $(\mathrm{s}), 144.7(\mathrm{~s}), 139.5$ (d), 139.3 (d), $111.3(\mathrm{~d}), 64.3(\mathrm{t}), 44.4(\mathrm{~d})$, $44.2(\mathrm{~d}), 42.2\left(\mathrm{t}_{2}\right), 26.9(\mathrm{~d}), 26.5(\mathrm{~d}), 21.0(\mathrm{q})$. 
Table 4. Study of the Leaving Group ${ }^{a}$

(d.r.)

${ }^{a}$ Reaction conditions: substrate $(0.5 \mathrm{mmol}), \mathrm{Na}\left(29 \mathrm{mg}, 1.25 \mathrm{mmol}, 2.5\right.$ equiv), $\mathrm{NH}_{3}(20 \mathrm{~mL}) .{ }^{b} 6$ equiv of Na were used.

Scheme 4. Postulated Reaction Mechanism for the Reductive Isomerization

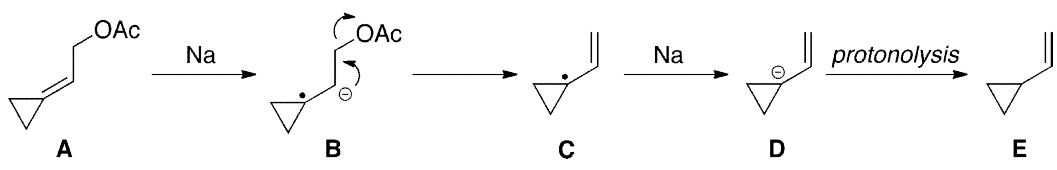

$(1 R *, 2 S *, 5 S *)$-Di-tert-butyl 3-(2-acetoxyethylidene)-6,7diazatricyclo[3.2.1.0 $\left.0^{2,4}\right]$ octane-6,7-dicarboxylate 16 . Following the general procedure $\mathrm{A}$, after purification on silica gel chromatography, the product was obtained as a white solid ( $1.46 \mathrm{~g}, 97 \%$ yield $)$. $\mathrm{Mp}=$ 84-85 ${ }^{\circ} \mathrm{C}$. ${ }^{1} \mathrm{H}$ NMR $\left(400 \mathrm{MHz}, \mathrm{CDCl}_{3}\right) \delta=5.90,(\mathrm{~m}, 1 \mathrm{H}), 4.82$ (br s, $1 \mathrm{H}), 4.58-4.72(\mathrm{~m}, 2 \mathrm{H}), 4.55($ br s, $1 \mathrm{H}), 2.08(\mathrm{~s}, 3 \mathrm{H}), 1.97$ (br s, $1 \mathrm{H}), 1.49(\mathrm{~s}, 18 \mathrm{H}), 1.18-1.36(\mathrm{~m}, 2 \mathrm{H}), 0.80-0.90(\mathrm{~m}, 1 \mathrm{H})$. ${ }^{13} \mathrm{C}$ NMR (100 MHz, $\left.\mathrm{CDCl}_{3}\right) \delta=170.5$ (s) 157.1 (br s), 156.1 (br s), $127.9(\mathrm{~s}), 115.8(\mathrm{~d}), 81.3(\mathrm{~s}, 2 \mathrm{C}), 63.5(\mathrm{t}), 62.0(\mathrm{~d}, 2 \mathrm{C}), 30.3(\mathrm{t})$, 28.0 (q, 6C), 20.7 (q), 19.4 (br d), 18.0 (br d). HRMS (ESI-MS) $m / z:[\mathrm{M}+\mathrm{H}]^{+}$, calcd for $\mathrm{C}_{20} \mathrm{H}_{31} \mathrm{~N}_{2} \mathrm{O}_{6}{ }^{+}, 395.2177$; found, 395.2173.

$\left(1 R^{*}, 25 *, 5 S *\right)$-Diethyl 3-(2-acetoxyethylidene)-6,7-diazatricyclo[3.2.1.0 ${ }^{2,4}$ ]octane-6,7-dicarboxylate 1c. Following the general procedure A, after purification on silica gel chromatography, the product was obtained as a colorless oil $\left(465 \mathrm{mg}, 71 \%\right.$ yield). ${ }^{1} \mathrm{H}$ $\operatorname{NMR}\left(400 \mathrm{MHz}, \mathrm{CDCl}_{3}\right) \delta=5.91,(\mathrm{~m}, 1 \mathrm{H}), 4.86(\mathrm{br} \mathrm{s}, 1 \mathrm{H}), 4.50-$ $4.74(\mathrm{~m}, 3 \mathrm{H}), 4.15-4.32(\mathrm{~m}, 4 \mathrm{H}), 2.06(\mathrm{~s}, 3 \mathrm{H}), 1.51(\mathrm{~d}, J=10.8$ $\mathrm{Hz}, 1 \mathrm{H}), 1.24-1.35(\mathrm{~m}, 8 \mathrm{H}), 0.81-0.93(\mathrm{~m}, 1 \mathrm{H}) .{ }^{13} \mathrm{C}$ NMR $(100$ $\mathrm{MHz}, \mathrm{CDCl}_{3}$ ) $\delta=170.7$ (s) 158.3 (br s, 2C), 127.7 (s), 116.2 (d), $63.6(\mathrm{t}), 62.5(\mathrm{t}, 2 \mathrm{C}), 62.5(\mathrm{br} \mathrm{d}, 2 \mathrm{C}), 30.9(\mathrm{~d}), 30.5(\mathrm{t}), 23.8(\mathrm{~d})$, 20.9 (q, 2C), 14.5 (q). HRMS (ESI-MS) $\mathrm{m} / z:[\mathrm{M}+\mathrm{H}]^{+}$, calcd for $\mathrm{C}_{16} \mathrm{H}_{23} \mathrm{~N}_{2} \mathrm{O}_{6}^{+}$, 339.1551; found, 339.1553 .

$2-((1 a S *, 2 S *, 2 a S *, 5 a S *, 6 R *)-2,2 a, 5,5 a, 6,6 a-H e x a h y d r o-2,6-$ methanocyclopropa[f]inden-1(1aH)-ylidene)ethyl Acetate 1d. Following the general procedure $\mathrm{A}$, after purification on silica gel chromatography, the product was obtained as a colorless oil (469 $\mathrm{mg}, 69 \%$ yield). The product was isolated as a mixture of 2 diastereomers. ${ }^{1} \mathrm{H}$ NMR $\left(400 \mathrm{MHz}, \mathrm{CDCl}_{3}\right) \delta=5.84(\mathrm{~m}, 1 \mathrm{H}), 5.67$ $(\mathrm{m}, 1 \mathrm{H}), 5.59(\mathrm{~m}, 1 \mathrm{H}), 4.54-4.67(\mathrm{~m}, 2 \mathrm{H}), 3.14(\mathrm{~m}, 1 \mathrm{H}), 2.58-$ $2.67(\mathrm{~m}, 1 \mathrm{H}), 2.49-2.55(\mathrm{~m}, 1 \mathrm{H}), 2.35-2.43(\mathrm{~m}, 1 \mathrm{H}), 2.21-2.35$ $(\mathrm{m}, 2 \mathrm{H}), 2.05(\mathrm{~s}, 3 \mathrm{H}), 1.44(\mathrm{~m}, 1 \mathrm{H}), 1.12-1.17(\mathrm{~m}, 1 \mathrm{H}), 1.10$ (pseudo d, $J=9.8 \mathrm{~Hz}, 1 \mathrm{H}$ ), 1.00 (pseudo d, $J=9.8 \mathrm{~Hz}, 1 \mathrm{H}) .{ }^{13} \mathrm{C}$ NMR $\left(100 \mathrm{MHz}, \mathrm{CDCl}_{3}\right)$ major diastereomer $\delta=170.9(\mathrm{~s}), 134.62$ (s), 131.78 (d), 131.40 (d), 113.9 (d), 64.6 (t), 53.6 (d), 42.22 (d), $41.3(\mathrm{~d}), 40.2(\mathrm{~d}), 33.0(\mathrm{t}), 31.86(\mathrm{t}), 21.0(\mathrm{q}), 18.5(\mathrm{~d}), 16.0$ (d); minor diastereomer $\delta=170.9(\mathrm{~s}), 134.59(\mathrm{~s}), 131.81(\mathrm{~d}), 131.35(\mathrm{~d})$, $113.9(\mathrm{~d}), 64.6(\mathrm{t}), 53.6(\mathrm{~d}), 42.20(\mathrm{~d}), 41.2(\mathrm{~d}), 40.3(\mathrm{~d}), 33.0(\mathrm{t})$, 31.90 (t), 21.0 (q), 18.8 (d), 15.6 (d). HRMS (ESI-MS) $\mathrm{m} / z$ : $[\mathrm{M}+$ $\mathrm{Na}]^{+}$, calcd for $\mathrm{C}_{15} \mathrm{H}_{18} \mathrm{O}_{2} \mathrm{Na}^{+}, 253.1199$; found, 253.1200.

$\left(1 R^{*}, 2 \mathrm{~S} * 5 \mathrm{~S} *, \mathrm{Z}\right)$-Dimethyl 3-(2-acetoxyethylidene)tricyclo[3.2.1.0 2,4 ]oct-6-ene-6,7-dicarboxylate 1e. Following the general procedure $\mathrm{A}$, after purification on silica gel chromatography, the product was obtained as a yellow oil (378 $\mathrm{mg}, 54 \%$ yield $).{ }^{1} \mathrm{H}(400$ $\left.\mathrm{MHz}, \mathrm{CDCl}_{3}\right) \delta 5.87(\mathrm{~m}, 1 \mathrm{H}), 4.60-4.72(\mathrm{~m}, 2 \mathrm{H}), 3.80(\mathrm{~s}, 3 \mathrm{H})$, $3.80(\mathrm{~s}, 3 \mathrm{H}), 3.44(\mathrm{~d}, J=6.5 \mathrm{~Hz}, 2 \mathrm{H}), 2.07(\mathrm{~s}, 3 \mathrm{H}), 1.92-1.99(\mathrm{~m}$, $2 \mathrm{H}), \quad 1.15-1.27(\mathrm{~m}, 2 \mathrm{H}) \cdot{ }^{13} \mathrm{C}\left(100 \mathrm{MHz}, \mathrm{CDCl}_{3}\right) \delta 170.8(\mathrm{~s})$, $165.29(\mathrm{~s}), 165.27(\mathrm{~s}), 148.3(\mathrm{~s}), 148.2(\mathrm{~s}), 141.0(\mathrm{~s}), 114.08(\mathrm{~d})$, $63.8(\mathrm{t}), 52.1(\mathrm{q}, 2 \mathrm{C}), 47.8(\mathrm{~d}), 47.7(\mathrm{~d}), 40.6(\mathrm{t}), 26.8(\mathrm{~d}), 26.6$ (d), 21.0 (d). HRMS (ESI-MS) $m / z:[\mathrm{M}+\mathrm{H}]^{+}$, calcd for $\mathrm{C}_{16} \mathrm{H}_{19} \mathrm{O}_{6}^{+}, 307.1177$; found, 307.1176 .

2-((1R*,2S*,5S*,6S*,7R*)-6,7-Bis(methoxymethyl)-8-oxatricyclo[3.2.1.0 $\left.{ }^{2,4}\right]$ octan-3-ylidene)ethyl Acetate if. Following the general 
procedure $\mathrm{A}$, after purification on silica gel chromatography, the product was obtained as a yellow oil (221 $\mathrm{mg}, 33 \%$ yield). ${ }^{1} \mathrm{H}$ NMR $\left(400 \mathrm{MHz}, \mathrm{CDCl}_{3}\right) \delta=5.90(\mathrm{~m}, 1 \mathrm{H}), 4.62(\mathrm{~m}, 2 \mathrm{H}), 4.48(\mathrm{~d}, J=9.0$ $\mathrm{Hz}, 2 \mathrm{H}) 3.33-3.36(\mathrm{~m}, 2 \mathrm{H}), 3.32(\mathrm{~s}, 6 \mathrm{H}), 3.19-3.26(\mathrm{~m}, 2 \mathrm{H})$, 2.25-2.40 (m, 2H), $2.04(\mathrm{~s}, 3 \mathrm{H}), 1.67-1.75(\mathrm{~m}, 2 \mathrm{H}) .{ }^{13} \mathrm{C}$ NMR $\left(100 \mathrm{MHz}, \mathrm{CDCl}_{3}\right) \delta=170.8(\mathrm{~s}), 128.5$ (s), $115.1(\mathrm{~d}), 79.9(\mathrm{~d}, 2 \mathrm{C})$, $70.7(\mathrm{t}, 2 \mathrm{C}), 64.1(\mathrm{t}), 58.8(\mathrm{q}, 2 \mathrm{C}), 46.2(\mathrm{~d}), 46.1(\mathrm{~d}), 22.2(\mathrm{q}), 21.7$ (d), 21.0 (d). HRMS (ESI-MS) $m / z:[\mathrm{M}+\mathrm{Na}]^{+}$, calcd for $\mathrm{C}_{15} \mathrm{H}_{22} \mathrm{O}_{5} \mathrm{Na}^{+}$, 305.1359; found, 305.1359 .

$2-((1 R *, 4 S *, 5 S *, 6 \mathrm{~S} *, 7 R *)-6,7-B i$ (cyanomethyl)-8-oxatricyclo[3.2.1.0 $0^{2,4}$ octan-3-ylidene)ethyl Acetate 1g. Following the general procedure A, after purification on silica gel chromatography, the product was obtained as a yellow oil (432 $\mathrm{mg}, 56 \%$ yield). ${ }^{1} \mathrm{H}$ NMR $\left(400 \mathrm{MHz}, \mathrm{CDCl}_{3}\right) \delta=5.97(\mathrm{tt}, J=1.2,6.4 \mathrm{~Hz}, 1 \mathrm{H}), 4.59-4.70(\mathrm{~m}$, $2 \mathrm{H}), 4.50(\mathrm{~d}, J=7.0 \mathrm{~Hz}, 2 \mathrm{H}), 2.52-2.59(\mathrm{~m}, 2 \mathrm{H}), 2.39-2.49(\mathrm{~m}$, $4 \mathrm{H}), 2.07(\mathrm{~s}, 3 \mathrm{H}), 1.81-1.88(\mathrm{~m}, 2 \mathrm{H}) .{ }^{13} \mathrm{C}$ NMR $(100 \mathrm{MHz}$, $\left.\mathrm{CDCl}_{3}\right) \delta=170.6(\mathrm{~s}), 125.4(\mathrm{~s}), 118.3(\mathrm{~s}, 2 \mathrm{C}), 116.8(\mathrm{~d}), 81.8(\mathrm{~d}$, 2C), $63.6(\mathrm{t}), 43.2$ (d), 43.1 (d), 21.5 (d), 21.2 (d), 20.9 (q), 17.0 $\left(\mathrm{t}, 2 \mathrm{C}\right.$ ). HRMS (ESI-MS) $\mathrm{m} / z:\left[\mathrm{M}+\mathrm{NH}_{4}\right]^{+}$, calcd for $\mathrm{C}_{15} \mathrm{H}_{20} \mathrm{~N}_{3} \mathrm{O}_{3}{ }^{+}$, 290.1499; found, 290.1502 .

1-Phenyl-2-((1R*,2S*,5S*)-tricyclo[3.2.1.0 $0^{2,4}$ ]octan-3-ylidene)ethyl Acetate $1 \mathrm{~h}$. Following the general procedure $\mathrm{A}$, the product was obtained as a colorless oil in a 1:1 mixture of 2 diastereomers and used without further purification $\left(510 \mathrm{mg}, 99 \%\right.$ yield). ${ }^{1} \mathrm{H}$ NMR $\left(400 \mathrm{MHz}, \mathrm{CDCl}_{3}\right) \delta=7.33-7.36(\mathrm{~m}, 4 \mathrm{H}), 7.28-7.31(\mathrm{~m}, 1 \mathrm{H})$, $6.35(\mathrm{t}, J=6.8 \mathrm{~Hz}, 1 \mathrm{H}), 5.89(\mathrm{tt}, J=1.2,6.5 \mathrm{~Hz}, 1 \mathrm{H}), 2.41(\mathrm{~m}$, $0.5 \mathrm{H}), 2.38(\mathrm{~m}, 0.5 \mathrm{H}), 2.35(\mathrm{~m}, 0.5 \mathrm{H}), 2.26(\mathrm{~m}, 0.5 \mathrm{H}), 2.11(\mathrm{~s}, 3 \mathrm{H})$, $1.45-1.53(\mathrm{~m}, 2 \mathrm{H}), 1.31-1.39(\mathrm{~m}, 2 \mathrm{H}), 1.18-1.29(\mathrm{~m}, 2 \mathrm{H}), 0.95$ $(\mathrm{dm}, J=9.8 \mathrm{~Hz}, 0.5 \mathrm{H}), 0.80(\mathrm{dm}, J=9.8 \mathrm{~Hz}, 0.5 \mathrm{H}), 0.76(\mathrm{dm}, J=$ $9.8 \mathrm{~Hz}, 0.5 \mathrm{H}), 0.70(\mathrm{dm}, J=9.8 \mathrm{~Hz}, 0.5 \mathrm{H}) .{ }^{13} \mathrm{C}$ NMR $(100 \mathrm{MHz}$, $\left.\mathrm{CDCl}_{3}\right) \delta 170.10(\mathrm{~s}), 170.07$ (s), $140.2(\mathrm{~s}), 140.1$ (s), $132.9(\mathrm{~s})$, 132.8 (s), 128.4 (d, 4C), 127.8 (d), 127.7 (d), 126.8 (d, 2C), 128.7 (d, 2C), 118.2 (d), 118.0 (d), 76.3 (d), 75.9 (d), 38.1 (d), 38.0 (d), $37.92(d), 37.86(d), 30.7(t), 30.6(t), 28.65(t), 28.59(t), 28.5(t$, 2C), 21.81 (d), 21.76 (d), 21.44 (d), 21.41 (d), 20.94 (d), 20.87 (d). HRMS (ESI-MS) $m / z:[\mathrm{M}+\mathrm{Na}]^{+}$, calcd for $\mathrm{C}_{18} \mathrm{H}_{20} \mathrm{O}_{2} \mathrm{Na}^{+}$, 291.1356; found, 291.1358 .

2-((1aS*,2S*,2aS*,5aS*,6R*)-2,2a,5,5a,6,6a-Hexahydro-2,6methanocyclopropa[f]inden-1(1aH)-ylidene)ethyl Benzoate $1 i$. Following the general procedure A, after purification on silica gel chromatography, the product was obtained as a colorless oil (408 $\mathrm{mg}, 80 \%$ yield). The product was isolated as a mixture of 2 diastereomers. ${ }^{1} \mathrm{H}$ NMR $\left(400 \mathrm{MHz}, \mathrm{CDCl}_{3}\right) \delta=8.03-8.09$ (br d, $2 \mathrm{H}), 7.52-7.59(\mathrm{~m}, 1 \mathrm{H}), 7.40-7.48(\mathrm{~m}, 2 \mathrm{H}), 5.93-6.00(\mathrm{~m}, 1 \mathrm{H})$, 5.66-5.72 (m, 1H), 5.57-5.63 (m, 1H), 4.82-4.94 (m, 2H), 3.11$3.19(\mathrm{~m}, 1 \mathrm{H}), 2.59-2.68(\mathrm{~m}, 1 \mathrm{H}), 2.51-2.56(\mathrm{~m}, 1 \mathrm{H}), 2.38-2.43$ $(\mathrm{m}, 1 \mathrm{H}), 2.21-2.38(\mathrm{~m}, 2 \mathrm{H}), 1.48(\mathrm{dd}, J=7.0,15.3 \mathrm{~Hz}, 1 \mathrm{H}), 1.12-$ $1.23(\mathrm{~m}, 2 \mathrm{H}), 1.00$ (pseudo $\mathrm{d}, J=10.0 \mathrm{~Hz}, 1 \mathrm{H}) .{ }^{13} \mathrm{C}$ NMR $(100$ $\left.\mathrm{MHz}, \mathrm{CDCl}_{3}\right)$ major diastereomer $\delta=166.4(\mathrm{~s}), 134.6(\mathrm{~s}), 132.7(\mathrm{~d})$, 131.7 (d), 131.4 (d), 130.5 (s), 129.5 (d, 2C), 128.2 (d, 2C), 113.9 (d), $65.0(\mathrm{t}), 53.6$ (d), 42.2 (d), $41.3(\mathrm{~d}), 40.2(\mathrm{~d}), 33.0(\mathrm{t}), 31.9$ (t), $18.6(\mathrm{~d}), 15.9(\mathrm{~d})$; partial minor diastereomer $\delta=131.8$ (d), 131.3 (d), 41.2 (d), 40.3 (d), 31.9 (t), 18.7 (d), 15.7 (d). HRMS (ESI-MS) $m / z:\left[\mathrm{M}+\mathrm{NH}_{4}\right]^{+}$, calcd for $\mathrm{C}_{20} \mathrm{H}_{24} \mathrm{NO}_{2}{ }^{+}, 310.1802$; found, 310.1799 .

$(2 R *, 2 a S *, 5 a S *, 6 S *, 6 a S *)-1-(2$-Phenoxyethylidene)$1,1 a, 2,2 a, 3,5 a, 6,6 a$-octahydro-2,6-methanocyclopropa[f]indene $1 j$. Following the general procedure $\mathrm{A}$, after purification on silica gel chromatography, the product was obtained as a colorless oil (288 $\mathrm{mg}, 62 \%$ yield). The product was isolated as a mixture of 2 diastereomers. ${ }^{1} \mathrm{H}$ NMR $\left(400 \mathrm{MHz}, \mathrm{CDCl}_{3}\right) \delta=7.24-7.30(\mathrm{~m}$, $2 \mathrm{H}), 6.88-6.95(\mathrm{~m}, 3 \mathrm{H}), 5.94-6.00(\mathrm{~m}, 1 \mathrm{H}), 5.66-5.72(\mathrm{~m}, 1 \mathrm{H})$, 5.63-5.75 (m, 1H), 4.54-4.66 (m, 2H), 3.11-3.19 (m, 1H), 2.59$2.68(\mathrm{~m}, 1 \mathrm{H}), 2.49-2.54(\mathrm{~m}, 1 \mathrm{H}), 2.22-2.41(\mathrm{~m}, 3 \mathrm{H}), 1.44-1.50$ $(\mathrm{m}, 1 \mathrm{H}), 1.13-1.20(\mathrm{~m}, 1 \mathrm{H}), 1.11$ (pseudo $\mathrm{d}, J=9.8 \mathrm{~Hz}, 1 \mathrm{H}), 1.00$ (pseudo d, $J=9.8 \mathrm{~Hz}, 1 \mathrm{H}) \cdot{ }^{13} \mathrm{C}$ NMR $\left(100 \mathrm{MHz}, \mathrm{CDCl}_{3}\right)$ major diastereomer $\delta=158.6(\mathrm{~s}), 133.7(\mathrm{~s}), 131.8(\mathrm{~d}), 131.4(\mathrm{~d}), 129.3(\mathrm{~d}$, 2C), 120.5 (d), 115.2 (d), 114.8 (d, 2C), 67.9 (t), 53.6 (d), 42.3 (d), $41.3(\mathrm{~d}), 40.1(\mathrm{~d}), 33.1(\mathrm{t}), 31.9(\mathrm{t}), 18.6(\mathrm{~d}), 16.1(\mathrm{~d})$; minor diastereomer (partial) $\delta=131.8$ (d), 131.4 (d), 129.4 (d, 2C), 41.1 (d), 40.3 (d), 18.9 (d), 15.7 (d). HRMS (ESI-MS) $m / z:[\mathrm{M}+\mathrm{Na}]^{+}$, calcd for $\mathrm{C}_{19} \mathrm{H}_{20} \mathrm{ONa}^{+}, 287.1406$; found, 287.1406.

$(2 R *, 2 a S *, 5 a S *, 6 S *, 6 a S *)-1-(2-($ Benzyloxy)ethylidene)$1,1 a, 2,2 a, 3,5 a, 6,6 a$-octahydro-2,6-methanocyclopropa[f]indene $1 \mathrm{k}$. Following the general procedure A, after purification on silica gel chromatography, the product was obtained as a colorless oil $(397 \mathrm{mg}$ $63 \%$ yield). The product was isolated as a mixture of 2 diastereomers. ${ }^{1} \mathrm{H}$ NMR $\left(400 \mathrm{MHz}, \mathrm{CDCl}_{3}\right) \delta=7.32-7.36(\mathrm{~m}$, $4 \mathrm{H}), 7.26-7.31(\mathrm{~m}, 1 \mathrm{H}), 5.90(\mathrm{~m}, 1 \mathrm{H}), 5.69(\mathrm{~m}, 1 \mathrm{H}), 5.60(\mathrm{~m}$, $1 \mathrm{H}), 4.48(\mathrm{~m}, 2 \mathrm{H}), 4.09(\mathrm{~m}, 2 \mathrm{H}), 3.15(\mathrm{~m}, 1 \mathrm{H}), 2.58-2.68(\mathrm{~m}$, $1 \mathrm{H}), 2.48-2.55(\mathrm{~m}, 1 \mathrm{H}), 2.32-2.42(\mathrm{~m}, 2 \mathrm{H}), 2.22-2.23(\mathrm{~m}, 1 \mathrm{H})$, $1.43(\mathrm{~m}, 1 \mathrm{H}), 1.09-1.15(\mathrm{~m}, 1 \mathrm{H}), 1.17$ (pseudo d, $J=9.8 \mathrm{~Hz}, 1 \mathrm{H})$, 1.02 (pseudo d, $J=9.8 \mathrm{~Hz}, 1 \mathrm{H}) .{ }^{13} \mathrm{C}$ NMR $\left(100 \mathrm{MHz}, \mathrm{CDCl}_{3}\right.$ ) major diastereomer $\delta=138.7(\mathrm{~s}), 132.9(\mathrm{~s}), 131.8(\mathrm{~d}), 131.4(\mathrm{~d})$, $128.3(\mathrm{~d}, 2 \mathrm{C}), 127.7$ (d, 2C), $127.4(\mathrm{~d}), 116.5(\mathrm{~d}), 71.5(\mathrm{t}), 70.2(\mathrm{t})$, 53.7 (d), 42.3 (d), $41.2(\mathrm{~d}), 40.2$ (d), $33.1(\mathrm{t}), 31.9(\mathrm{t}), 18.3$ (d), 16.0 (d); minor diastereomer (partial) $\delta=131.5$ (d), 53.6 (d), 42.3 (d), 40.1 (d), 18.8 (d), 15.4 (d). HRMS (ESI-MS) $m / z:[\mathrm{M}+\mathrm{Li}]^{+}$, calcd for $\mathrm{C}_{20} \mathrm{H}_{22} \mathrm{OLi}^{+}$, 285.1826; found, 285.1819.

2-Methyl-1-((1R*,4S*,5S*)-tricyclo[3.2.1.0.2,4]oct-6-en-3-ylidene)propan-2-ol 11. ${ }^{11}$ Following the general procedure A, after purification on silica gel chromatography, the product was obtained as a colorless oil $\left(531 \mathrm{mg}, 99 \%\right.$ yield). ${ }^{1} \mathrm{H}$ NMR $\left(400 \mathrm{MHz}, \mathrm{CDCl}_{3}\right)$ $\delta=6.36(\mathrm{~s}, 2 \mathrm{H}), 5.80(\mathrm{~s}, 1 \mathrm{H}), 3.00(\mathrm{~m}, 2 \mathrm{H}), 1.79(\mathrm{br} \mathrm{s}, 1 \mathrm{H}), 1.64$ (pseudo d, $J=7.8 \mathrm{~Hz}, 1 \mathrm{H}$ ), 1.46 (pseudo $\mathrm{d}, J=7.3,1 \mathrm{H}), 1.38(\mathrm{~s}$, $6 \mathrm{H}), 1.07(\mathrm{~d}, J=8.5 \mathrm{~Hz}, 1 \mathrm{H}), 0.92(\mathrm{~d}, J=8.3 \mathrm{~Hz}, 1 \mathrm{H}) .{ }^{13} \mathrm{C}$ NMR $\left(100 \mathrm{MHz}, \mathrm{CDCl}_{3}\right) \delta 139.4$ (d), 138.9 (d), 135.8 (s), 125.0 (d), 71.7 (s), 44.2 (d), 44.0 (d), $41.8(\mathrm{t}), 29.8(\mathrm{q}), 29.7$ (q), 27.2 (d), 24.0 (d). HRMS (ESI-MS) $m / z:[\mathrm{M}+\mathrm{Na}]^{+}$, calcd for $\mathrm{C}_{12} \mathrm{H}_{16} \mathrm{ONa}^{+}$, 199.1093; found, 199.1093.

$\left(1 R^{*}, 2 \mathrm{~S} *, 5 \mathrm{~S} *\right)-3-\left(\right.$ Benzylidene)tricyclo[3.2.1.0. $\left.0^{2,4}\right]$ octane $1 \mathrm{~m} .^{11}$ Following the general procedure $\mathrm{A}$, after purification on silica gel chromatography, the product was obtained as a colorless oil (649 $\mathrm{mg}, 86 \%$ yield). ${ }^{1} \mathrm{H}$ NMR ( $\left.400 \mathrm{MHz}, \mathrm{CDCl}_{3}\right) \delta=7.46-7.51$ (m, $2 \mathrm{H}), 7.28-7.35(\mathrm{~m}, 2 \mathrm{H}), 7.16-7.22(\mathrm{~m}, 1 \mathrm{H}), 6.63(\mathrm{~s}, 1 \mathrm{H}), 2.61-$ $2.65(\mathrm{~m}, 1 \mathrm{H}), 2.48-2.52(\mathrm{~m}, 1 \mathrm{H}), 1.55-1.65(\mathrm{~m}, 3 \mathrm{H}), 1.42-1.52$ $(\mathrm{m}, 2 \mathrm{H}), 1.34(\mathrm{~d}, \mathrm{~J}=7.3 \mathrm{~Hz}, 1 \mathrm{H}), 1.01(\mathrm{~d}, \mathrm{~J}=9.9 \mathrm{~Hz}, 1 \mathrm{H}), 0.81(\mathrm{~d}$, $\mathrm{J}=9.9 \mathrm{~Hz}, 1 \mathrm{H}) \cdot{ }^{13} \mathrm{C} \mathrm{NMR}\left(100 \mathrm{MHz}, \mathrm{CDCl}_{3}\right) \delta=138.1(\mathrm{~s}), 130.7$ (s),128.4 (d), 126.5 (d), 126.4 (d), 119.5 (d), 38.4 (d), 38.0 (d), $30.9(\mathrm{t}), 28.9(\mathrm{t}), 28.6(\mathrm{t}), 23.1(\mathrm{~d}), 19.5(\mathrm{~d})$.

2-((1aS*,2S*,2aS*,5aS*,6R*)-2,2a,5,5a,6,6a-Hexahydro-2,6methanocyclopropa[f]inden-1(1aH)-ylidene)ethanol $1 \mathrm{n}$. To a solution of $1 \mathrm{~d}(230 \mathrm{mg}, 1 \mathrm{mmol})$ in $\mathrm{MeOH}(10 \mathrm{~mL})$ was added $\mathrm{K}_{2} \mathrm{CO}_{3}$ (690 mg, $5 \mathrm{mmol}, 5$ equiv) at room temperature. The reaction was followed by TLC, and after $2 \mathrm{~h}$, the conversion was completed. The reaction mixture was evaporated to dryness, and the residue was dissolved with $\mathrm{Et}_{2} \mathrm{O}(10 \mathrm{~mL})$ and $\mathrm{H}_{2} \mathrm{O}(10 \mathrm{~mL})$. The product was extracted with $\mathrm{Et}_{2} \mathrm{O}(3 \times 10 \mathrm{~mL})$, and the combined organic layers were dried over anhydrous $\mathrm{Na}_{2} \mathrm{SO}_{4}$. The solvents were removed under reduced pressure, and after purification on silica gel chromatography, the product was obtained as a colorless oil (141 $\mathrm{mg}, 75 \%$ yield). The product was isolated as a mixture of 2 diastereomers. ${ }^{1} \mathrm{H}\left(400 \mathrm{MHz}, \mathrm{CDCl}_{3}\right) \delta=5.94(\mathrm{~m}, 1 \mathrm{H}), 5.68(\mathrm{~m}$, $1 \mathrm{H}), 5.51(\mathrm{~m}, 1 \mathrm{H}), 4.20(\mathrm{~m}, 2 \mathrm{H}), 3.14(\mathrm{~m}, 1 \mathrm{H}), 2.58-2.68(\mathrm{~m}$, $1 \mathrm{H}), 2.51(\mathrm{~m}, 1 \mathrm{H}), 2.20-2.40(\mathrm{~m}, 3 \mathrm{H}), 1.35-1.48(\mathrm{~m}, 2 \mathrm{H}), 1.08-$ $1.19(\mathrm{~m}, 2 \mathrm{H}), 1.01$ (pseudo $\mathrm{d}, J=9.8 \mathrm{~Hz}, 1 \mathrm{H}) .{ }^{13} \mathrm{C}$ NMR $(100$ $\mathrm{MHz}, \mathrm{CDCl}_{3}$ ) major diastereomer $\delta=131.9(\mathrm{~s}), 131.8(\mathrm{~d}), 131.4(\mathrm{~d})$, $118.9(\mathrm{~d}), 63.2(\mathrm{t}), 53.6(\mathrm{~d}), 42.3(\mathrm{~d}), 41.2(\mathrm{~d}), 40.11(\mathrm{~d}), 32.9(\mathrm{t})$, $31.8(\mathrm{t}), 18.1(\mathrm{~d}), 15.0(\mathrm{~d})$; minor diastereomer (partial) $\delta=17.8(\mathrm{~d})$, 15.2 (d). HRMS (ESI-MS) $m / z:[\mathrm{M}+\mathrm{Na}]^{+}$, calcd for $\mathrm{C}_{13} \mathrm{H}_{16} \mathrm{ONa}^{+}, 211.1093$; found, 211.1094.

2-((1R*,2R*,4S*,5S*,8R*)-3-Oxatetracyclo[3.3.1.0.0 $\left.0^{2,4} \cdot 0^{6,8}\right]$ nonan7-ylidene)ethyl Acetate 10. A solution of meta-chloroperbenzoic acid (50-55\% purity, $1 \mathrm{~g}, 2.90 \mathrm{mmol}, 1.1$ equiv) in $13 \mathrm{~mL}$ of dry DCM was added slowly at $-20{ }^{\circ} \mathrm{C}$ in $40 \mathrm{~min}$ to a solution of cycloadduct $1 \mathrm{a}(500 \mathrm{mg}, 2.63 \mathrm{mmol})$ in $13 \mathrm{~mL}$ of dry DCM. The mixture was stirred at $-20{ }^{\circ} \mathrm{C}$ for $24 \mathrm{~h}$ and let to reach slowly $0{ }^{\circ} \mathrm{C}$. After basic hydrolysis with aqueous $\mathrm{Na}_{2} \mathrm{CO}_{3}$ solution and extraction with DCM, the organic phase was washed with brine and dried over $\mathrm{Na}_{2} \mathrm{SO}_{4}$. The white crude solid was purified by flash chromatography 
on silica gel to afford the expected product as a pale oil $(426 \mathrm{mg}$, $79 \%$ yield $) .{ }^{1} \mathrm{H}\left(400 \mathrm{MHz}, \mathrm{CDCl}_{3}\right) \delta=5.85(\mathrm{tt}, J=1.3,6.5 \mathrm{~Hz}$, $1 \mathrm{H}), 4.56-4.57(\mathrm{~m}, 2 \mathrm{H}), 3.38-3.42(\mathrm{~m}, 2 \mathrm{H}), 2.65-2.71(\mathrm{~m}, 2 \mathrm{H})$, $2.06(\mathrm{~s}, 3 \mathrm{H}), 1.53-1.60(\mathrm{~m}, 2 \mathrm{H}), 0.99(\mathrm{~d}, J=10.4, \mathrm{~Hz}, 1 \mathrm{H}), 0.53$ $(\mathrm{d}, J=10.4 \mathrm{~Hz}, 1 \mathrm{H}) \cdot{ }^{13} \mathrm{C}\left(100 \mathrm{MHz}, \mathrm{CDCl}_{3}\right) \delta=170.8(\mathrm{~s}), 134.1$ $(\mathrm{s}), 115.1(\mathrm{~d}), 63.9(\mathrm{t}), 54.8$ (d, 2C), 38.8 (d), 38.7 (d), 21.0 (d), 20.6 (q), 20.3 (d), $17.2(\mathrm{t})$. HRMS (ESI-MS) $\mathrm{m} / z:[\mathrm{M}+\mathrm{Na}]^{+}$, calcd for $\mathrm{C}_{12} \mathrm{H}_{14} \mathrm{O}_{3} \mathrm{Na}^{+}, 229.0835$; found, 229.0835 .

$(1 R *, 2 S *, 5 S *)$-Di-tert-butyl 3-(2-Hydroxyethylidene)-6,7diazatricyclo[3.2.1.0 $0^{2,4}$ octane-6,7-dicarboxylate $1 p$. Following the general procedure $\mathrm{A}$, after purification on silica gel chromatography, the product was obtained as a white solid $(703 \mathrm{mg}, 83 \%$ yield $) . \mathrm{Mp}$ $=142-144{ }^{\circ} \mathrm{C} .{ }^{1} \mathrm{H}\left(400 \mathrm{MHz}, \mathrm{CDCl}_{3}\right) \delta=6.00(\mathrm{~m}, 1 \mathrm{H}), 4.82(\mathrm{br} \mathrm{s}$, $1 \mathrm{H}), 4.56(\mathrm{br} \mathrm{s}, 1 \mathrm{H}), 4.25(\mathrm{br} \mathrm{d}, J=5.3,2 \mathrm{H}), 1.62-2.03(\mathrm{~m}, 3 \mathrm{H})$, $1.49(\mathrm{~s}, 18 \mathrm{H}), 1.20-1.33(\mathrm{~m}, 2 \mathrm{H}) .{ }^{13} \mathrm{C}$ NMR $\left(100 \mathrm{MHz}, \mathrm{CDCl}_{3}\right) \delta$ $=156.8($ br s, 2C), $125.0(\mathrm{~s}), 120.8(\mathrm{~d}), 81.6(\mathrm{~s}, 2 \mathrm{C}), 62.5(\mathrm{t}), 62.2$ (br d, 2C), $30.4(\mathrm{t}), 28.1$ (q, 6C), 19.3 (br d), 17.8 (br d). HRMS (ESI-MS) $m / z:[\mathrm{M}+\mathrm{H}]^{+}$, calcd for $\mathrm{C}_{18} \mathrm{H}_{29} \mathrm{~N}_{2} \mathrm{O}_{5}{ }^{+}, 353.2071$; found, 353.2071.

General Procedure B: Reductive Isomerization. In a Schlenk flask equipped with a gas condenser and cooled at $-60{ }^{\circ} \mathrm{C}$ was condensed $10 \mathrm{~mL}$ of ammonia. The methylene cyclopropane derivative 1 ( $1 \mathrm{mmol}$ ) was dissolved in $3 \mathrm{~mL}$ of THF (or $\mathrm{Et}_{2} \mathrm{O}$ for volatile adduct) and then introduced into the Schlenk. Sodium (58 mg, $2.5 \mathrm{mmol}$ ) was added, resulting in an immediate dark blue coloration. After almost $30 \mathrm{~min}$, the solution turned pale yellow, indicating total consumption of free sodium radical. Solid $\mathrm{NH}_{4} \mathrm{Cl}(50$ $\mathrm{mg}$ ) was then added, and ammonia was evaporated by slowly warming the flask at room temperature. After addition of water ( 5 $\mathrm{mL})$, the crude product was extracted with AcOEt $(3 \times 5 \mathrm{~mL})$ and purified over silica.

$\left(1 R^{*}, 2 R^{*}, 5 \mathrm{~S}^{*}\right)$-Di-tert-butyl 3-Vinyl-6,7-diazatricyclo[3.2.1.0 $\left.0^{2,4}\right]$ octane-6,7-dicarboxylate $5 b$. Following the general procedure $\mathrm{B}$, after purification on silica gel chromatography, the product was obtained as a white solid $(221 \mathrm{mg}, 66 \%$ yield $) . \mathrm{Mp}=73-75{ }^{\circ} \mathrm{C} .{ }^{1} \mathrm{H}$ NMR (400 MHz, $\mathrm{CDCl}_{3}$ ) $\delta=5.25$ (ddd, $J=8.3,10.0,17.1 \mathrm{~Hz}$, $1 \mathrm{H}), 5.08(\mathrm{dd}, J=1.5,17.1 \mathrm{~Hz}, 1 \mathrm{H}), 4.93(\mathrm{dd}, J=1.5,10.0 \mathrm{~Hz}$, $1 \mathrm{H}), 4.78($ br s, $1 \mathrm{H}), 4.53(\mathrm{br} \mathrm{s}, 1 \mathrm{H}), 1.49(\mathrm{~s}, 18 \mathrm{H}), 1.35-1.50(\mathrm{~m}$, $4 \mathrm{H}), 1.11-1.25(\mathrm{~m}, 1 \mathrm{H}) .{ }^{13} \mathrm{C}$ NMR $\left(100 \mathrm{MHz}, \mathrm{CDCl}_{3}\right) \delta=157.2$ (br s, 2C), 136.8 (d), 114.0 (t), 81.2 (s, 2C), 61.0 (br d, 2C), 28.0 (q, 6C), $22.5($ br d), $21.1(\mathrm{~d}, 2 \mathrm{C})$, one $\mathrm{C}(\mathrm{t})$ is missing. HRMS (ESI-MS) $m / z:[\mathrm{M}+\mathrm{H}]^{+}$, calcd for $\mathrm{C}_{18} \mathrm{H}_{29} \mathrm{~N}_{2} \mathrm{O}_{4}{ }^{+}, 337.2122$; found, 337.2119 .

$(1 R, * 2 R *, 5 S *)-6,7-B i s\left(\right.$ ethylperoxy)-3-vinyltricyclo[3.2.1.0. $\left.0^{2,4}\right]$ oct-6ene $5 \mathrm{C}$. Following the general procedure $\mathrm{B}$, after purification on silica gel chromatography, the product was obtained as a colorless oil (154 mg, $55 \%$ yield). ${ }^{1} \mathrm{H}$ NMR $\left(400 \mathrm{MHz}, \mathrm{CDCl}_{3}\right) \delta=5.22$ (ddd, $J=8.3$, $10.0,17.1 \mathrm{~Hz}, 1 \mathrm{H}), 5.06(\mathrm{dd}, J=1.5,17.1 \mathrm{~Hz}, 1 \mathrm{H}), 4.91(\mathrm{dd}, J=$ $1.3,10.3 \mathrm{~Hz}, 1 \mathrm{H}), 4.80$ (br s, $1 \mathrm{H}), 4.58$ (br s, $1 \mathrm{H}), 4.21$ (br s, $4 \mathrm{H})$, $1.48-1.53(\mathrm{~m}, 1 \mathrm{H}), 1.45(\mathrm{~d}, J=11.5 \mathrm{~Hz}, 1 \mathrm{H}), 1.27(\mathrm{t}, J=6.8 \mathrm{~Hz}$, $6 \mathrm{H}), 1.17-1.25(\mathrm{~m}, 3 \mathrm{H}) .{ }^{13} \mathrm{C} \mathrm{NMR}\left(100 \mathrm{MHz}, \mathrm{CDCl}_{3}\right) \delta=158.4$ (br s, 2C), 136.6 (d), 114.5 (t), 62.4 (t, 2C), 61.3 (br d, 2C), 28.3 (t), 21.8 (d), 21.2 (d, 2C), 14.4 (q, 2C). HRMS (ESI-MS) $\mathrm{m} / \mathrm{z}$ : [M $+\mathrm{H}]^{+}$, calcd for $\mathrm{C}_{14} \mathrm{H}_{21} \mathrm{~N}_{2} \mathrm{O}_{4}{ }^{+}, 281.1496$; found, 281.1496.

$\left(2 R *, 2 a S^{*}, 5 a S^{*}, 6 S^{*}, 6 a R^{*}\right)-1$-Vinyl-1, 1a,2,2a,3,5a,6,6a-octahydro-2,6-methanocyclopropa[f]indene $5 d$. Following the general procedure $\mathrm{B}$, after purification on silica gel chromatography, the product was obtained as a 10:1 mixture of diastereomers (148 mg, $86 \%$ yield). ${ }^{1} \mathrm{H}$ NMR $\left(400 \mathrm{MHz}, \mathrm{CDCl}_{3}\right)$ major diastereomer $\delta=$ 5.68-5.74 (m, $1 \mathrm{H}), 5.52-5.58(\mathrm{~m}, 1 \mathrm{H}), 5.26(\mathrm{ddd}, J=9.0,10.0$, $17.1 \mathrm{~Hz}, 1 \mathrm{H}), 5.00(\mathrm{dd}, J=1.8,17.1 \mathrm{~Hz}, 1 \mathrm{H}), 4.79(\mathrm{dd}, J=1.8,10.0$ $\mathrm{Hz}, 1 \mathrm{H}), 3.04-3.12(\mathrm{~m}, 1 \mathrm{H}), 2.49-2.58(\mathrm{~m}, 1 \mathrm{H}), 2.39-2.44(\mathrm{~m}$, $1 \mathrm{H}), 2.27-2.34(\mathrm{~m}, 2 \mathrm{H}), 2.16-2.26(\mathrm{~m}, 1 \mathrm{H}), 1.48(\mathrm{dt}, J=9.0,2.5$ $\mathrm{Hz}, 1 \mathrm{H}), 1.12(\mathrm{~d}, J=10.3 \mathrm{~Hz}, 1 \mathrm{H}), 0.85-0.93(\mathrm{~m}, 2 \mathrm{H}), 0.59-0.66$ $(\mathrm{m}, 1 \mathrm{H})$; partial minor diastereomer $\delta=6.05(\mathrm{ddd}, J=4.5,10.3,17.3$ $\mathrm{Hz}, 1 \mathrm{H}), 5.64-5.68(\mathrm{~m}, 1 \mathrm{H}), 5.46-5.51(\mathrm{~m}, 1 \mathrm{H}), 5.14(\mathrm{dm}, J=$ $17.3 \mathrm{~Hz}, 1 \mathrm{H}) .{ }^{13} \mathrm{C}\left(100 \mathrm{MHz}, \mathrm{CDCl}_{3}\right)$ major diastereomer $\delta=140.9$ (d), $132.2(\mathrm{~d}), 130.8$ (d), $111.4(\mathrm{t}), 54.6$ (d), 43.0 (d), 39.5 (d), $38.2(\mathrm{~d}), 31.4(\mathrm{t}), 31.3(\mathrm{t}), 22.0(\mathrm{~d}), 19.0(\mathrm{~d}), 17.3(\mathrm{~d}) ;$ minor diastereomer $\delta=137.6(\mathrm{~d}), 131.8(\mathrm{~d}), 131.5(\mathrm{~d}), 116.0(\mathrm{t}), 52.2(\mathrm{~d})$,
43.6 (d), 39.4 (d), 38.4 (d), 33.5 (t), $31.6(\mathrm{t}), 20.0$ (d), 19.5 (d), 16.6 (d). HRMS (ESI-MS) $m / z:[\mathrm{M}+\mathrm{H}]^{+}$, calcd for $\mathrm{C}_{13} \mathrm{H}_{17}{ }^{+}$, 173.1325; found, 173.1325 .

$(1 R *, 2 R *, 5 S *, 6 S *, 7 R *)-6,7-B i s$ (methoxymethyl)-3-vinyl-8oxatricyclo[3.2.1. $\left.0^{2,4}\right]$ octane $5 f$. Following the general procedure $\mathrm{B}$, after purification on silica gel chromatography, the product was obtained as a 10:1 mixture of diastereomers (143 mg, 64\% yield). ${ }^{1} \mathrm{H}$ NMR $\left(400 \mathrm{MHz}, \mathrm{CDCl}_{3}\right)$ major diastereomer $\delta=5.36$ (ddd, $J=9.0$, $10.3,17.1 \mathrm{~Hz}, 1 \mathrm{H}), 5.03(\mathrm{dd}, J=1.2,17.1 \mathrm{~Hz}, 1 \mathrm{H}), 4.85(\mathrm{dd}, J=$ $1.3,10.3 \mathrm{~Hz}, 1 \mathrm{H}), 4.28(\mathrm{~s}, 2 \mathrm{H}), 3.31(\mathrm{~s}, 6 \mathrm{H}), 3.29-3.33(\mathrm{~m}, 2 \mathrm{H})$, $3.18-3.24(\mathrm{~m}, 2 \mathrm{H}), 2.15-2.21(\mathrm{~m}, 2 \mathrm{H}), 1.55(\mathrm{dt}, J=2.8,8.8 \mathrm{~Hz}$, $1 \mathrm{H}), 1.14(\mathrm{~d}, J=2.8 \mathrm{~Hz}, 2 \mathrm{H})$; partial minor diastereomer $\delta=6.17$ (ddd, $J=7.3,10.3,17.3 \mathrm{~Hz}, 1 \mathrm{H}), 5.10(\mathrm{dd}, J=1.6,17.3 \mathrm{~Hz}, 1 \mathrm{H})$, $4.96(\mathrm{dd}, J=1.6,10.3 \mathrm{~Hz}, 1 \mathrm{H}), 4.41(\mathrm{~s}, 3 \mathrm{H}) .{ }^{13} \mathrm{C}(100 \mathrm{MHz}$, $\left.\mathrm{CDCl}_{3}\right)$ major diastereomer $\delta=137.5(\mathrm{~d}), 113.0(\mathrm{t}), 78.6(\mathrm{~d}, 2 \mathrm{C})$, 70.7 (t, 2C), 58.7 (q, 2C), 46.5 (d, 2C), 24.1 (d, 2C), 21.3 (d); partial minor diastereomer $\delta=135.7(\mathrm{~d}) 115.4(\mathrm{t}), 78.5(\mathrm{~d}, 2 \mathrm{C}), 58.7$ (q, 2C), 46.8 (d, 2C), 23.1 (d, 2C), 21.3 (d). HRMS (ESI-MS) $\mathrm{m} / \mathrm{z}$ : $[\mathrm{M}+\mathrm{H}]^{+}$, calcd for $\mathrm{C}_{13} \mathrm{H}_{21} \mathrm{O}_{3}{ }^{+}, 225.1485$; found, 225.1484 .

$2,2^{\prime}-\left(\left(1 R^{*}, 4 R^{*}, 5 S^{*}, 6 \mathrm{~S} *, 7 R^{*}\right)-3-\right.$ Vinyl-8-oxatricyclo[3.2.1.0 $\left.0^{2,4}\right]$ octane-6,7-diyl)diacetonitrile $5 \mathrm{~g}$. Following the general procedure $\mathrm{B}$, after purification on silica gel chromatography, the product was obtained as a $3.3: 1$ mixture of diastereomers $(53 \mathrm{mg}$, $25 \%$ yield). Pale yellow solid. $\mathrm{Mp}=103-105{ }^{\circ} \mathrm{C} .{ }^{1} \mathrm{H}$ NMR $\left(400 \mathrm{MHz}, \mathrm{CDCl}_{3}\right)$ major diastereomer $\delta=5.25$ (ddd, $J=8.8,10.3,17.1 \mathrm{~Hz}, 1 \mathrm{H}), 5.01$ (dd, $J=1.0,17.1 \mathrm{~Hz}, 1 \mathrm{H}), 4.87(\mathrm{dd}, J=1.5,10.3 \mathrm{~Hz}, 1 \mathrm{H}), 4.26(\mathrm{~s}$, $2 \mathrm{H}), 2.37-2.45(\mathrm{~m}, 6 \mathrm{H}), 1.58$ (ddd, $J=2.8,3.0,8.5 \mathrm{~Hz}, 1 \mathrm{H}), 1.21$ $(\mathrm{d}, J=2.8 \mathrm{~Hz}, 2 \mathrm{H})$; partial minor diastereomer $\delta=6.05-5.96-(\mathrm{m}$, $1 \mathrm{H}), 5.09(\mathrm{dd}, J=1.8,17.6 \mathrm{~Hz}, 1 \mathrm{H}), 4.96(\mathrm{dd}, J=2.0,10.5 \mathrm{~Hz}$, $1 \mathrm{H}) .{ }^{13} \mathrm{C}$ NMR $\left(100 \mathrm{MHz}, \mathrm{CDCl}_{3}\right)$ major diastereomer $\delta=135.9$ (d), $118.4(\mathrm{~s}, 2 \mathrm{C}), 114.4(\mathrm{t}), 80.8(\mathrm{~d}, 2 \mathrm{C}), 43.6(\mathrm{~d}, 2 \mathrm{C}), 23.6$ (d, 2C), $18.2(\mathrm{~d}), 17.0(\mathrm{t}, 2 \mathrm{C})$; partial minor diastereomer $\delta=134.0(\mathrm{~d})$, $118.4(\mathrm{~s}), 116.9(\mathrm{t}), 80.9(\mathrm{~d}, 2 \mathrm{C}), 43.8(\mathrm{~d}, 2 \mathrm{C}), 22.7(\mathrm{~d}, 2 \mathrm{C})$. HRMS (ESI-MS) $m / z:\left[\mathrm{M}+\mathrm{NH}_{4}\right]^{+}$, calcd for $\mathrm{C}_{13} \mathrm{H}_{18} \mathrm{~N}_{3} \mathrm{O}^{+}$, 232.1444; found, 232.1444 .

$\left(1 R^{*}, 2 S *, 5 S *\right)-3-((E)$-styryl)tricyclo[3.2.1.0.0 2,4 octane (minor) $5 \mathrm{~h}$ and $\left(1 R^{*}, 2 R^{*}, 5 S^{*}\right)$-3-Phenethyltricyclo[3.2.1.0 $0^{2,4}$ ]octane (major) $7 h$. Following the general procedure $B$, after purification on silica gel chromatography, products were obtained as an inseparable mixture $(157 \mathrm{mg}, 12 \%$ yield for $5 \mathrm{~h}$ and $62 \%$ yield for $7 \mathrm{~h}) .{ }^{1} \mathrm{H}$ NMR $\left(400 \mathrm{MHz}, \mathrm{CDCl}_{3}\right)$ major product $\delta=7.24-7.31(\mathrm{~m}, 2 \mathrm{H}), 7.14-$ $7.20(\mathrm{~m}, 3 \mathrm{H}), 2.65(\mathrm{dd}, J=7.5,8.0 \mathrm{~Hz}, 2 \mathrm{H}), 2.24(\mathrm{br} \mathrm{s}, 2 \mathrm{H}), 1.33-$ $1.44(\mathrm{~m}, 4 \mathrm{H}), 1.18-1.25(\mathrm{~m}, 2 \mathrm{H}), 0.97-0.99(\mathrm{~m}, 1 \mathrm{H}), 0.66-0.73$ $(\mathrm{m}, 1 \mathrm{H}), 0.57-0.62(\mathrm{~m}, 1 \mathrm{H}), 0.43-0.48(\mathrm{~m}, 2 \mathrm{H})$; partial minor product $\delta=6.41(\mathrm{~d}, J=15.8 \mathrm{~Hz}, 1 \mathrm{H}), 5.68(\mathrm{dd}, J=9.3,15.8 \mathrm{~Hz}$, $1 \mathrm{H}), 2.37$ (br s, $2 \mathrm{H}), 1.83-1.89(\mathrm{~m}, 1 \mathrm{H}), 1.12-1.18(\mathrm{~m}, 1 \mathrm{H}), 1.01-$ $1.08(\mathrm{~m}, 1 \mathrm{H}) .{ }^{13} \mathrm{C}$ NMR $\left(100 \mathrm{MHz}, \mathrm{CDCl}_{3}\right)$ major product $\delta=$ 142.7 (s), 128.4 (d, 2C), 128.1 (d, 2C), 125.5 (d), 36.0 (d, 2C), 35.9 $(\mathrm{t}), 34.0(\mathrm{t}), 29.7(\mathrm{t}, 2 \mathrm{C}), 28.6(\mathrm{t}), 22.9(\mathrm{~d}, 2 \mathrm{C}), 13.7(\mathrm{~d})$; partial minor product $\delta=137.8(\mathrm{~s}), 132.8(\mathrm{~d}), 128.2(\mathrm{~d}), 127.1$ (d), 126.4 (d), 36.3 (d), $30.3(\mathrm{t}), 29.4$ (t), 25.0 (d), 17.8 (d). HRMS (ESI-MS) major product $m / z$ : $\left[\mathrm{M}+\mathrm{NH}_{4}\right]^{+}$, calcd for $\mathrm{C}_{16} \mathrm{H}_{24} \mathrm{~N}^{+}, 230.1903$; found, 230.1904 .

$\left(1 R^{*}, 4 S^{*}, 5 S^{*}\right)-3-(2-M e t h y l p r o p-1-e n-1-y \mid)$ tricyclo[3.2.1.02,4 $]$ oct-6ene 51 . Following the general procedure $\mathrm{B}$, after purification on silica gel chromatography, the product obtained as a 6:1 mixture of diastereomers (69 mg, 43\% yield). ${ }^{1} \mathrm{H}$ NMR (400 $\mathrm{MHz}, \mathrm{CDCl}_{3}$ ) major diastereomer $\delta=6.35-6.38(\mathrm{~m}, 2 \mathrm{H}), 4.61-4.66(\mathrm{dm}, J=9.3$ $\mathrm{Hz}, 1 \mathrm{H}), 2.84(\mathrm{br} \mathrm{s}, 2 \mathrm{H}), 2.55(\mathrm{dt}, J=9.5,2.5 \mathrm{~Hz}, 1 \mathrm{H}), 1.74(\mathrm{~d}, J=$ $1.0 \mathrm{~Hz}, 3 \mathrm{H}), 1.66(\mathrm{~d}, J=1.0 \mathrm{~Hz}, 3 \mathrm{H}), 1.22(\mathrm{dm}, J=9.3 \mathrm{~Hz}, 1 \mathrm{H})$, $0.95(\mathrm{~d}, J=2.3 \mathrm{~Hz}, 2 \mathrm{H}), 0.88(\mathrm{dm}, J=9.3 \mathrm{~Hz}, 1 \mathrm{H})$; partial minor diastereomer $\delta=6.50(\mathrm{~m}, 2 \mathrm{H}), 1.71(\mathrm{dm}, J=5.5 \mathrm{~Hz}, 2 \mathrm{H}), 1.17(\mathrm{dm}$, $J=7.3 \mathrm{~Hz}, 1 \mathrm{H}) .{ }^{13} \mathrm{C} \mathrm{NMR}\left(100 \mathrm{MHz}, \mathrm{CDCl}_{3}\right)$ major diastereomer $\delta$ $=140.4(\mathrm{~d}, 2 \mathrm{C}), 129.5(\mathrm{~s}), 125.9(\mathrm{~d}) 41.8(\mathrm{~d}, 2 \mathrm{C}), 38.8(\mathrm{t}), 31.5$ (d), $30.3(\mathrm{~d}, 2 \mathrm{C}), 25.5(\mathrm{q}), 18.3(\mathrm{q})$; minor diastereomer $\delta=142.2$ (d, 2C), 133.2 (s), 122.3 (d), 42.2 (d, 2C), 41.5 (t), 37.0 (d), 28.7 (d, 2C), 25.2 (q), 18.2 (q). HRMS (ESI-MS) $\mathrm{m} / z:[\mathrm{M}+\mathrm{Ag}]^{+}$, calcd for $\mathrm{C}_{12} \mathrm{H}_{16} \mathrm{Ag}^{+}, 267.0297$; found, 267.0298 .

$\left(1 R^{*}, 2 R^{*}, 4 S^{*}, 5 S^{*}, 8 R^{*}\right)-7-$ Vinyl-3-oxatetracyclo[3.3.1.0 $\left.0^{2,4} \cdot 0^{6,8}\right]$ nonane 50 . Following the general procedure $\mathrm{B}$, after purification on 
silica gel chromatography, the product obtained as a colorless oil (146 mg, 99\% yield). ${ }^{1} \mathrm{H}$ NMR $\left(400 \mathrm{MHz}, \mathrm{CDCl}_{3}\right) \delta=5.31$ (ddd, $J$ $=8.5,10.3,17.0 \mathrm{~Hz}, 1 \mathrm{H}), 5.01(\mathrm{dd}, J=1.5,17.0 \mathrm{~Hz}, 1 \mathrm{H}), 4.83(\mathrm{dd}$, $J=1.5,10.3 \mathrm{~Hz}, 1 \mathrm{H}), 3.32(\mathrm{~s}, 2 \mathrm{H}), 2.60(\mathrm{~s}, 2 \mathrm{H}), 1.94(\mathrm{dt}, J=8.5$, $1.6 \mathrm{~Hz}, 1 \mathrm{H}$ ), 1.00 (d, $J=2.5 \mathrm{~Hz}, 2 \mathrm{H}), 0.96$ (pseudo d, $J=11,0 \mathrm{~Hz}$, $1 \mathrm{H}), 0.57$ (pseudo $\mathrm{d}, J=11.0 \mathrm{~Hz}, 1 \mathrm{H}) .{ }^{13} \mathrm{C}\left(100 \mathrm{MHz}, \mathrm{CDCl}_{3}\right) \delta$ $138.6(\mathrm{~d}), 112.4(\mathrm{t}), 55.0$ (d, 2C), 37.1 (d, 2C), 23.8 (d, 2C), 23.5 (d), $14.7(\mathrm{t})$. HRMS (ESI-MS) $\mathrm{m} / z$ : $[\mathrm{M}+\mathrm{H}]^{+}$, calcd for $\mathrm{C}_{10} \mathrm{H}_{13} \mathrm{O}^{+}$, 149.0961; found, 149.0963 .

Di-tert-butyl $\left(\left(1 R^{*}, 2 R^{*}, 4 S^{*}\right)\right.$-6-vinylbicyclo[3.1.0]hexane-2,4diyl)dicarbamate $6 b$. Following the general procedure B, after purification on silica gel chromatography, the product obtained as a white solid (186 mg, 55\% yield). Mp $=144-146{ }^{\circ} \mathrm{C} .{ }^{1} \mathrm{H}$ NMR (400 $\left.\mathrm{MHz}, \mathrm{CDCl}_{3}\right) \delta=5.29$ (ddd, $\left.J=8.5,10.3,17.1 \mathrm{~Hz}, 1 \mathrm{H}\right), 4.96(\mathrm{dd}, J$ $=1.3,17.1 \mathrm{~Hz}, 1 \mathrm{H}), 4.83(\mathrm{dd}, J=1.3,10.3 \mathrm{~Hz}, 1 \mathrm{H}), 4.01(\mathrm{br} \mathrm{s}, 2 \mathrm{H})$, $1.72-1.85(\mathrm{~m}, 1 \mathrm{H}), 1.61(\mathrm{~d}, J=15.6 \mathrm{~Hz}, 1 \mathrm{H}), 1.52(\mathrm{~m}, 2 \mathrm{H}), 1.47$ $(\mathrm{m}, 2 \mathrm{H}), 1.43(\mathrm{~s}, 18 \mathrm{H}), 1.04(\mathrm{~m}, 1 \mathrm{H}) .{ }^{13} \mathrm{C}$ NMR $(100 \mathrm{MHz}$, $\left.\mathrm{CDCl}_{3}\right) \delta=155.4$ (s, 2C), 137.8 (d), $112.9(\mathrm{t}), 79.3$ (br s, 2C), 52.3 (br d, 2C), 38.3 (t), 38.3 (d), 28.4 (q, 6C), 23.4 (d, 2C). HRMS (ESI-MS) $m / z:[\mathrm{M}+\mathrm{H}]^{+}$, calcd for $\mathrm{C}_{18} \mathrm{H}_{31} \mathrm{~N}_{2} \mathrm{O}_{4}^{+}, 339.2278$; found, 339.2277 .

$\left(1 R^{*}, 2 R *, 5 S *\right)-3-$ Benzyltricyclo[3.2.1.0 $\left.0^{2,4}\right]$ octane $7 m$. Following the general procedure $\mathrm{B}$, after purification on silica gel chromatography, the product obtained as a colorless oil (196 mg, 99\% yield). ${ }^{1} \mathrm{H}$ NMR (400 MHz, $\left.\mathrm{CDCl}_{3}\right) \delta=7.18-7.34(\mathrm{~m}, 5 \mathrm{H}), 2.43(\mathrm{~d}, J=$ $7.0 \mathrm{~Hz}, 2 \mathrm{H}), 2.30(\mathrm{br} \mathrm{s}, 2 \mathrm{H}), 1.41-1.47(\mathrm{~m}, 2 \mathrm{H}), 1.23-1.30(\mathrm{~m}$, $2 \mathrm{H}), 0.95-1.03(\mathrm{~m}, 2 \mathrm{H}), 0.61-0.67(\mathrm{~m}, 3 \mathrm{H}) .{ }^{13} \mathrm{C} \mathrm{NMR}(100 \mathrm{MHz}$, $\left.\mathrm{CDCl}_{3}\right) \delta=142.3(\mathrm{~s}), 128.3(\mathrm{~d}, 2 \mathrm{C}), 128.2(\mathrm{~d}, 2 \mathrm{C}), 125.7(\mathrm{~d}), 37.7$ $(\mathrm{t}), 36.0(\mathrm{~d}, 2 \mathrm{C}), 29.6(\mathrm{t}, 2 \mathrm{C}), 28.6(\mathrm{t}), 23.2(\mathrm{~d}, 2 \mathrm{C}), 14.8(\mathrm{~d})$. HRMS (ESI-MS) $m / z:[\mathrm{M}+\mathrm{Ag}]^{+}$, calcd for $\mathrm{C}_{15} \mathrm{H}_{18} \mathrm{Ag}^{+}, 305.0454$; found, 305.0451 .

\section{AUTHOR INFORMATION}

\section{Corresponding Author}

*E-mail: herve.clavier@univ-amu.fr

Notes

The authors declare no competing financial interest.

\section{ACKNOWLEDGMENTS}

This work was supported by Centrale Marseille, the CNRS and AMU. We gratefully acknowledge Dr. Michel Giorgi, Dr. Valérie Monnier and Christophe Chendo (Spectropole, Fédération des Sciences Chimiques de Marseille) for respectively the $\mathrm{X}$-ray analysis and MS analyses.

\section{REFERENCES}

(1) For reviews on vinylcyclopropanes, see: (a) Rubin, M.; Rubina, M.; Gevorgyan, V. Chem. Rev. 2007, 107, 3117-3179. (b) Hudlicky, T.; Reed, J. W. Angew. Chem., Int. Ed. 2010, 49, 4864-4876. (c) Jiao, L.; Yu, Z.-X. J. Org. Chem. 2013, 78, 6842-6848.

(2) For reviews, see: (a) Donaldson, W. A. Tetrahedron 2001, 57, 8589-8627. (b) Chen, D. Y.-K; Pouwer, R. H.; Richard, J.-A. Chem. Soc. Rev. 2012, 41, 4631-4642. For selected examples, see: (c) Liu, P.; Jacobsen, E. N. J. Am. Chem. Soc. 2001, 123, 10772-10773. (d) Yue, G.; Yang, L.; Yuan, C.; Du, B.; Liu, B. Tetrahedron 2012, $68,9624-9637$.

(3) For selected examples, see: (a) Jarstfer, M. B.; Blagg, B. S. J.; Rogers, D. H.; Poulter, C. D. J. Am. Chem. Soc. 1996, 118, 1308913090. (b) Larock, R. C.; Yum, E. K. Tetrahedron 1996, 52, $2743-$ 2758. (c) Adams, L. A.; Aggarwal, V. K.; Bonnert, R. V.; Bressel, B.; Cox, R. J.; Shepherd, J.; de Vicente, J.; Walter, M.; Whittingham, W. G.; Winn, C. L. J. Org. Chem. 2003, 68, 9433-9440. (d) Zhang, J.-L.; Chan, P. W. H.; Che, C. M. Tetrahedron Lett. 2003, 44, 8733-8737. (e) Jiang, H.; Sun, X.-L; Zhu, C.-Y.; Dai, L.-X.; Tang, Y. Tetrahedron 2008, 64, 5032-5035. (f) Barluenga, J.; Quiñones, N.; TomásGamasa, M.; Cabal, M.-P. Eur. J. Org. Chem. 2012, 2012, 2312-2317. (g) Yang, Y.; Li, J.; Du, B.; Yuan, C.; Liu, B.; Qin, S. Chem. Commun. 2015, 51, 6179-6182.

(4) (a) Craig, D.; Gore, S. J.; Lansdell, M. I.; Lewis, S. E.; Mayweg, A. V. W.; White, A. J. P. Chem. Commun. 2010, 46, 4991-4993. (b) Chang, M.-Y.; Chen, Y.-C.; Chan, C.-K. Tetrahedron 2014, 70, 8908-8913.

(5) (a) Pyun, H.-J.; Chaudhary, K.; Somoza, J. R.; Sheng, X. C.; Kim, C. U. Tetrahedron Lett. 2009, 50, 3833-3835. (b) Zhu, B.-H.; Zhou, R.; Zheng, J.-C.; Deng, X.-M.; Sun, X.-L.; Shen, Q.; Tang, Y. J. Org. Chem. 2010, 75, 3454-3457.

(6) For selected recent references, see: (a) Zhang, H.; Curran, D. P. J. Am. Chem. Soc. 2011, 133, 10376-10378. (b) Liang, Y.; Jiang, X.; Yu, Z.-X. Chem. Commun. 2011, 47, 6659-6661. (c) Tang, W.; Wei, X.; Yee, N. K.; Patel, N.; Lee, H.; Savoie, J.; Senanayake, C. H. Org. Process Res. Dev. 2011, 15, 1207-1211. (d) Rassadin, V. A.; Sokolov, V. V.; Khlebnikov, A. F.; Ulin, N. V.; Kozhushkov, S. I.; de Meijere, A. Synthesis 2012, 44, 372-376. (e) Hay, E. B.; Zhang, H.; Curran, D. P. J. Am. Chem. Soc. 2015, 137, 322-327.

(7) (a) Catellani, M.; Chiusoli, G. P. J. Organomet. Chem. 1982, 233, C21-C24. (b) Catellani, M.; Chiusoli, G. P. J. Organomet. Chem. 1983, 250, 509-515.

(8) Miura, T.; Sasaki, T.; Harumashi, T.; Murakami, M. J. Am. Chem. Soc. 2006, 128, 2516-2517.

(9) Tseng, N.-W.; Mancuso, J.; Lautens, M. J. Am. Chem. Soc. 2006, $128,5338-5339$.

(10) Achard, T.; Lepronier, A.; Gimbert, Y.; Clavier, H.; Giordano, L.; Tenaglia, A.; Buono, G. Angew. Chem., Int. Ed. 2011, 50, 35523556.

(11) Bigeault, J.; Giordano, L.; De Riggi, I.; Gimbert, Y.; Buono, G. Org. Lett. 2007, 9, 3567-3570.

(12) (a) Bigeault, J.; Giordano, L.; Buono, G. Angew. Chem., Int. Ed. 2005, 44, 4753-4757. (b) Bigeault, J.; de Riggi, I.; Gimbert, Y.; Giordano, L.; Buono, G. Synlett 2008, 2008, 1071-1075. (c) Thota, R.; Lesage, D.; Gimbert, Y.; Giordano, L.; Humbel, S.; Milet, A.; Buono, G.; Tabet, J.-C. Organometallics 2009, 28, 2735-2743. (d) Gatineau, D.; Moraleda, D.; Naubron, J.-V.; Bürgi, T.; Giordano, L.; Buono, G. Tetrahedron: Asymmetry 2009, 20, 1912-1917. (e) Clavier, H.; Lepronier, A.; Bengobesse-Mintsa, N.; Gatineau, D.; Pellissier, H.; Giordano, L.; Tenaglia, A.; Buono, G. Adv. Synth. Catal. 2013, 355, 403-408.

(13) (a) Ge, G.-C.; Mo, D.-L.; Ding, C.-H.; Dai, L.-X.; Hou, X.-L. Org. Lett. 2012, 14, 5756-5759. (b) Mo, D.-L.; Yuan, T.; Ding, C.H.; Dai, L.-X.; Hou, X.-L. J. Org. Chem. 2013, 78, 11470-11476.

(14) For the preparation of platinum-phosphinito-phosphinous acid complexes and their application in catalysis, see: (a) Bigeault, J.; Giordano, L.; de Riggi, I.; Gimbert, Y.; Buono, G. Org. Lett. 2007, 9, 3567-3570. (b) Achard, T.; Giordano, L.; Tenaglia, A.; Gimbert, Y.; Buono, G. Organometallics 2010, 29, 3936-3950.

(15) (a) Mellor, J. M.; Smith, M. N. J. Chem. Soc., Perkin Trans. 1 1984, 2927-2931. (b) Denmark, S. E.; Nicaise, O.; Edwards, J. P. J. Org. Chem. 1990, 55, 6219-6223. (c) Storsberg, J.; Nandakumar, M. V.; Sankaranarayanana, S.; Kaufmann, D. E. Adv. Synth. Catal. 2001, 343, 177-180. (d) Bournaud, C.; Robic, D.; Bonin, M.; Micouin, L. J. Org. Chem. 2005, 70, 3316-3317.

(16) For a review on bicyclic hydrazines, see: (a) Bournaud, C.; Chung, F.; Pérez Luna, A.; Pasco, M.; Errasti, G.; Lecourt, T.; Micouin, L. Synthesis 2009, 2009, 869-887. For alternative methods 
of N-N cleavage of bicyclic hydrazine, see: (b) Pérez Luna, A.; Ceschi, M.-A.; Bonin, M.; Micouin, L.; Husson, H.-P. J. Org. Chem. 2002, 67, 3522-3524. (c) Bournaud, C.; Bonin, M.; Micouin, L. Org. Lett. 2006, 8, 3041-3043. (d) Moumné, R.; Pasco, M.; Prost, E.; Lecourt, T.; Micouin, L.; Tisné, C. J. Am. Chem. Soc. 2010, 132, 13111-13113. (e) Pasco, M.; Moumné, R.; Lecourt, T.; Micouin, L. J. Org. Chem. 2011, 76, 5137-5142. (f) Blond, A.; Moumné, R.; Bégis, G.; Pasco, M.; Lecourt, T.; Micouin, L. Tetrahedron Lett. 2011, 52, 3201-3203. (g) Blond, A.; Dockerty, P.; Álvarez, R; Turcaud, S.; Lecourt, T.; Micouin, L. J. Org. Chem. 2013, 78, 12236-12242.

(17) For a rare example of $\mathrm{C}-\mathrm{O}$ bond reduction using $\mathrm{Na}$ in $\mathrm{NH}_{3}$, see: Birch, A. J. J. Chem. Soc. 1945, 809-813.

(18) For reviews on methylidenecyclopropanes, see: (a) Brandi, A.; Goti, A. Chem. Rev. 1998, 98, 589-636. (b) Audran, G.; Pellissier, H. Adv. Synth. Catal. 2010, 352, 575-608. (c) Pellissier, H. Tetrahedron 2010, 66, 8341-8375. (d) Brandi, A.; Cicchi, S.; Cordero, F. M.; Goti, A. Chem. Rev. 2014, 114, 7317-7420. (e) Pellissier, H. Tetrahedron 2014, 70, 4991-5031.

(19) (a) Bouveault, L.; Blanc, G. C. R. Hebd. Seances Acad. Sci. 1903, 136, 1676-1678. (b) Bouveault, L.; Blanc, G. Bull. Soc. Chim. 1904, 31, 666-672.

(20) Scott, N. D.; Walker, J. F.; Hansley, V. L. J. Am. Chem. Soc. 1936, 58, 2442-2444.

(21) (a) Metz, P. Tetrahedron 1989, 45, 7311-7316. (b) Marchand, A. P.; Reddy, G. M. Synthesis 1991, 1991, 198-200.

(22) See Supporting Information.

(23) Perrin, D. D.; Armarego, W. L. F. Purification of Laboratory Chemicals, 3rd ed.; Pergamon Press: Oxford, U.K., 1988.

(24) Fulmer, G. R.; Miller, A. J. M.; Sherden, N. H.; Gottlieb, H. E.; Nudelman, A.; Stoltz, B. M.; Bercaw, J. E.; Golberg, K. I. Organometallics 2010, 29, 2176-2179. 